\title{
The mixology of precursory strain partitioning approaching brittle failure in rocks
}

\author{
J. McBeck, ${ }^{1}$ Y. Ben-Zion ${ }^{2}$ and F. Renard ${ }^{\oplus 1,3}$ \\ ${ }^{1}$ Physics of Geological Processes, The Njord Centre, Department of Geosciences, University of Oslo, Oslo, Norway. E-mail: j.a.mcbeck@geo.uio.no \\ ${ }^{2}$ Department of Earth Sciences, University of Southern California, Los Angeles, CA, USA \\ ${ }^{3}$ Université Grenoble Alpes, Université Savoie Mont Blanc, CNRS, IRD, IFSTTAR, ISTerre, Grenoble, France
}

Accepted 2020 March 11. Received 2020 March 5; in original form 2019 October 22

\begin{abstract}
SUMMAR Y
We examine the strain accumulation and localization process throughout 12 triaxial compression experiments on six rock types deformed in an X-ray transparent apparatus. In each experiment, we acquire 50-100 tomograms of rock samples at differential stress steps during loading, revealing the evolving 3-D distribution of X-ray absorption contrasts, indicative of density. Using digital volume correlation (DVC) of pairs of tomograms, we build time-series of 3-D incremental strain tensor fields as the rocks are deformed towards failure. The Pearson correlation coefficients between components of the local incremental strain tensor at each stress step indicate that the correlation strength between pairs of local strain components, including dilation, contraction and shear strain, are moderate-strong in 11 of 12 experiments. In addition, changes in the local strain components from one DVC calculation to the next show differences in the correlations between pairs of strain components. In particular, the correlation of the local changes in dilation and shear strain tends to be stronger than the correlation of changes in dilation-contraction and contraction-shear strain. In 11 of 12 experiments, the most volumetrically frequent mode of strain accommodation includes a synchronized increase in multiple strain components. Early in loading, under lower differential stress, the most frequent strain accumulation mode involves the paired increase in dilation and contraction at neighbouring locations. Under higher differential stress, the most frequent mode is the paired increase in dilation and shear strain. This mode is also the first or second most frequent throughout each complete experiment. Tracking the mean values of the strain components in the sample and the volume of rock that each component occupies reveals fundamental differences in the nature of strain accumulation and localization between the volumetric and shear strain modes. As the dilative strain increases in magnitude throughout loading, it tends to occupy larger volumes within the rock sample and thus delocalizes. In contrast, the increasing shear strain components (left- or right-lateral) do not necessarily occupy larger volumes and so involve localization. Consistent with these evolutions, the correlation length of the dilatational strains tends to increase by the largest amounts of the strain components from lower to higher differential stress. In contrast, the correlation length of the shear strains does not consistently increase or decrease with increasing differential stress.
\end{abstract}

Key words: Creep and deformation; Fault zone rheology; Fracture and flow; Dynamics and mechanics of faulting; Fractures, faults, and high strain deformation zones.

\section{INTRODUCTION}

Recognizing precursory signals approaching the onset of macroscopic failure is a critical goal in rock mechanics. Experimental observations indicate that fracture coalescence leading to macroscopic failure occurs through the opening of individual fractures that interact to allow shear deformation on a macroscopic scale (e.g. Peng \& Johnson 1972; Petit \& Barquins 1988; Moore \& Lockner
1995). Consequently, much previous work has highlighted the importance of dilation as a precursor to brittle failure (e.g. Brace et al. 1966). Although evolving fractures may accommodate both opening and shearing, as wing-cracks for example (e.g. Wong \& Chau 1998), experimental and geophysical research has tended to focus on the dilatational deformation rather than the shear deformation because previous studies could not readily quantify the magnitudes of local dilation and shear strain operating within intact material or 
along individual fractures. Because the contribution of local dilation can influence the macroscopic expression of radial dilation, density and seismic velocities in expected ways, experimental methods could estimate the impact of local dilatational events (e.g. Bridgman 1949; Handin et al. 1963; Frank 1965; Brace et al. 1966; Myachkin et al. 1972). Fewer analyses have quantified local deformation mechanisms that accommodate shear strain in triaxial experiments within intact rock by resolving the source types of acoustic emission events (e.g. Stanchits et al. 2006), and tracking macroscopic stress-strain relations, seismic anisotropy and non-linear resonance during deformation (e.g. Hamiel et al. 2004a, 2009; Lyakhovsky et al. 2009).

Here, we characterize the strain accumulation and localization processes as rocks approach macroscopic failure, and compare the importance of contraction, dilation and shear strain in accommodating brittle deformation at varying stages of deformation. We analyse results from 12 triaxial compression deformation experiments on six rock types with an X-ray transparent deformation apparatus. In each experiment, we compress the rock core in stress steps and acquire a 3-D tomogram at each step, revealing the 3-D distribution of X-ray absorption. Digital volume correlation (DVC) analysis implemented in the code TomoWarp2 (Tudisco et al. 2017) provide the local 3-D displacement vectors, between tomogram acquisitions, that is the incremental displacement field. From the time-series of the incremental displacement fields, we calculate the contracting, dilating and shearing components of the incremental strain tensor. From the time-series of strain fields, we quantify how the incremental volumetric and shear strain components evolve during the approach to macroscopic failure.

This data set enables comparing the strain localization and accumulation process in experiments on sandstone, basalt, monzonite, granite, shale and limestone. We quantify the spatial correlation between each strain component, such as dilation and shear strain, and systematically compare the evolving correlations among pairs of strain components as well as different experiments and rock types. We track the strength of the strain accumulation process with the mean value of the strain component in each DVC calculation. We track the localization of the strain field using the volume of rock that the strain component occupies in each DVC calculation. We compare the accumulation and localization process for the volumetric strain components (dilation and contraction) and shear strain components (left- or right-lateral). We track the correlation length of each of the strain components in order to assess the growing (or shrinking) interaction distance of each strain component.

The results provide novel descriptions of the strain accumulation and localization processes in brittle rocks when approaching failure under the stress conditions of the upper crust. The correlation between the changes of dilation and shear strain from one tomogram to the next (or stress step) are the strongest of all the correlations between pairs of strain components. Tracking the evolving strain components reveals that the most frequent strain accommodation mode, in 11 of 12 experiments, is the paired increase of dilation and shear strain. Tracking the mean of each strain component and volume of rock occupied by the strain component reveals different behaviours of the volumetric and shear strains. Whereas dilation tends to occupy larger volumes (delocalizing) as it gains strength, shear strain tends to occupy the same or smaller volumes (localizing) as it gains strength. Tracking the correlation distance for each of the strain components throughout the loading shows that the correlation distance of the dilation increases by the most throughout loading compared to the other strain components.

\section{BRITTLE FAILURE PROCESSES IN ROCKS}

Characterizing the micromechanical processes that produce brittle failure began with theoretical solutions for the stress field surrounding circular and elliptical holes (Kolosov 1909; Inglis 1913). Griffith (1921) initiated the field of linear elastic fracture mechanics (LEFM) by developing an energy-based condition for fracture propagation that led to the concept of the stress intensity factor, $K$, at a fracture tip (Irwin 1948). Then, the stress field surrounding a crack tip could be approximated from the loading and stress intensity factors associated with tensile and shear fractures (e.g. Lawn 1993). There are several well-recognized limitations of this seminal work. The analytical formulations predict unbounded stresses at the crack tip because LEFM formulations do not incorporate inelastic deformation beyond a stress threshold. Fracture mechanics models that include inelastic deformation in a process zone that eliminate crack tip singularities (e.g. Rice 1980) help to address this limitation. Moreover, these analytical formulations predict the stress field surrounding a fracture tip and the likelihood of fracture propagation in systems with only one preexisting fracture or weakness that is much larger than other flaws in the system.

The Mohr-Coulomb failure criterion provides a macroscopic approach to understand brittle failure (Coulomb 1776; Mohr 1900), in contrast to the fracture-centric, stress intensity factor approach. This failure criterion uses the stress state and material properties of the rock to predict whether and at what orientation a new fault develops, or whether slip occurs along a pre-existing fault plane. Under low compressive stresses, these predictions statistically match observations (e.g. Mitra 1994; Storti et al. 1997; Crider \& Pollard 1998; McBeck et al. 2017). But under tensile conditions, the theoretical uniaxial tensile strength derived from this criterion tends to exceed experimental measurements (e.g. Paul 1961). The breakdown of the Mohr-Coulomb failure criterion in the tensile range leads to using two sets of failure criteria depending on whether the deformation is assumed to be dominated by tensile or shear deformation. These two sets of criteria produce, in turn, two different sets of stress thresholds for failure in tension or shear and two sets of predicted fracture geometries (e.g. Cooke \& Madden 2014).

Local instabilities, such as fracture growth, that produce macroscopic failure events may be understood by examining the conditions that lead to the localization of deformation (Rudnicki \& Rice 1975; Rice \& Rudnicki 1980; Lyakhovsky et al. 1997, 2011). Localization may occur at the critical conditions when the continuum equations of the increments of deformation lose ellipticity, or when the strain energy function of the material loses convexity. These and other analytical formulations are based on homogeneous solids, and so do not provide testable predictions about the precursors to macroscopic failure in heterogeneous solids such as rocks. Consequently, they do not provide insight into how to recognize the approach to brittle instabilities and macroscopic failures. Rock deformation experiments under triaxial compression show that systematic precursors to rock failure are detectable, at least in the laboratory (e.g. Reches \& Lockner 1994).

One of the most recognized precursors to failure in laboratory experiments is the macroscopic dilation of the rock sample. Monitoring the macroscopic axial and transverse strain of deformed rocks indicates that low porosity crystalline rocks dilate as they are compressed (e.g. Brace et al. 1966). The evolving reduction of seismic wave velocities and increasing $V_{p} / V_{s}$ ratios in experiments provides evidence for dilation as increasing volumes of air- or water-filled fractures develop (Nur 1972). Resolving the source types of acoustic emission events (e.g. Stanchits et al. 2006) further supports the 
concept that local tensile failure produces opening-mode deformation that leads to macroscopic dilation, as inferred from changes in the macroscopic strain and seismic velocities. Categorizing acoustic emissions into tensile events and mixed-mode or shear events during brittle deformation indicates that tensile failure can dominate the early deformation process, and that increasing proportions of mixedmode and shear events occur towards failure (e.g. Stanchits et al. 2006; Rodríguez \& Celestino 2019). These works highlight that rock failure includes a mixture of deformation modes. Moreover, both the criteria used for tensile failure (Griffith) and shear failure (MohrCoulomb) do not consider volume changes in their original formulations, although some analyses have worked to incorporate dilation into the Mohr-Coulomb framework (e.g. Hamiel et al. 2005; Zhao \& Cai 2010).

Deriving the source type of acoustic emissions indicates that compactive mechanisms can comprise 5-15 per cent of the deformation mode in both granite and basalt, even under low differential stress (20 MPa) (Stanchits et al. 2006). Cataclastic deformation bands with lower porosity than the surrounding rock (Aydin \& Johnson 1983) provide further evidence of local compaction as a dominant deformation mechanism. In porous rocks like basalt, sandstone and limestone that experience pore-collapse, local compaction may accommodate a greater proportion of the deformation than shear or dilation under certain stress conditions (e.g. Huang et al. 2019). Theoretical work that describes the conditions of pore collapse, such as the Hertzian fracture concept, focus on the local stress concentrations that develop at the edges of pores and between grains (e.g. Wong et al. 1997). Consequently, several diverging failure criteria describe the conditions of compactive, tensile and shear failure, although field and experimental evidence indicate that deformation occurs through the mixture of these modes (e.g. Thompson et al. 2005; Stanchits et al. 2006; Owladeghaffari 2015; Rodríguez \& Celestino 2019).

Experimental data from in situ X-ray microtomography experiments on rocks under triaxial deformation can provide key observations on the importance of dilatational, contractive and shear deformation mechanisms in different rocks under various stress and temperature conditions. Microtomography scans image the 3-D distribution of density contrasts produced by opening or closing fractures and pores, and minerals and rock fragments of different densities. Patterns of grey-level values that arise from density contrasts enable DVC on pairs of microtomography scans. DVC on pairs of scans acquired throughout triaxial rock deformation provide a time-series of the 3-D incremental strain tensor at varying differential stresses. The evolving statistics of the populations of dilatational, contractive and shear strains throughout the triaxial deformation enable comparing the relative importance of these deformation modes among different rock types. In addition, DVC provides both the seismic and aseismic components of deformation, whereas monitoring acoustic emissions only provides the detectable seismic events. Because DVC does not allow separating the aseismic and seismic components, we may not directly compare this work to previous analyses using acoustic emissions.

DVC analysis on Fontainebleau sandstone (Renard et al. 2019a) and Mount Etna basalt (McBeck et al. 2019) indicate that shear and dilation dominated the deformation process in these porous rocks under low confining stress (10-20 MPa). The mean of the dilatational strain population increased by larger magnitudes than the shear strain throughout these experiments, suggesting the greater importance of the micromechanisms that produce local dilation rather than shear. Tracking the 2-D strain field on the surface of Carrara marble loaded in uniaxial compression also reveals the dominance of shear and dilatational strain events throughout loading, with a greater frequency of dilatational events near macroscopic failure (Tal et al. 2016). DVC analyses on laminated Green River shale (McBeck et al. 2018) provide a different partitioning of the strain modes than observed in the experiments on marble, sandstone and basalt. In two experiments on shale, the magnitude of radial dilation was of the same order as the magnitude of axial contraction throughout loading (McBeck et al. 2018). Numerical modelling and the spatial distribution of localizing strain fields suggest that localized contraction within a subhorizontal volume promotes shear strain localization that leads to macroscopic failure of the shale (McBeck et al. 2018).

The varying partitioning of the strain modes in these different rock types prompts the current investigation of strain partitioning in 12 experiments with six different rock types. This work quantitatively compares the relative contribution of contraction, dilation and shear strain in triaxial deformation experiments on sandstone, basalt, monzonite, granite, shale and limestone. Such a comprehensive comparison of the strain accumulation and localization process has not yet been presented, and constitutes the major contribution of this work. Our X-ray tomography data, and accompanying DVC analysis, provide the full aseismic and seismic deformation field, whereas analyses of acoustic emissions are limited to only the seismic activity that produce detected emissions.

In particular, this work examines the synchronicity of the strain accumulation and localization process by finding the volume of rock occupied by increasing values of one, two or three of the strain modes (compaction, dilation and shear) at each stage of each experiment. The study links the strain accumulation (changing magnitude) and strain localization (changing volume) processes in the (contractive and dilative) volumetric and (left- and right-lateral) shear strain modes. The results may help to determine the appropriate failure criterion to use when predicting rock failure in varying differential stress conditions. The results may also help to indicate whether different rock types require different failure criteria to robustly predict the onset of macroscopic failure.

\section{METHODS}

\subsection{In situ X-ray tomography experiment conditions}

We deform rocks in the X-ray transparent deformation apparatus, HADES (Renard et al. 2016) installed on beamline ID19 at the European Synchrotron and Radiation Facility at room temperature. The rock samples are cylinders $1 \mathrm{~cm}$ in length and $4-5 \mathrm{~mm}$ in diameter (Table 1). In each experiment, we increase the axial stress in steps of $0.5-5 \mathrm{MPa}$ under constant confining stress between 5 and $35 \mathrm{MPa}$ (Table 1) until the sample fails macroscopically. At each axial stress step, we acquire a 3-D X-ray tomogram at $6.5 \mu \mathrm{m}$ per voxel-side resolution while the sample is under constant load inside the deformation apparatus. Each scan requires about $2 \mathrm{~min}$, and the final scan immediately precedes the macroscopic rock failure. Under larger confining stresses, the 2-min scan time could provide sufficient time for brittle creep. The quality of the scan indicates whether such deformation occurs because if the rock is moving while being scanned, the scan quality is poor. The high quality of the scans used in these experiments suggest that such deformation did not occur while the rocks were being scanned under constant load. We use stress-control loading conditions instead of strain-rate control to avoid sample motion during a scan. Due to the stress-controlled loading, the rock cores fail with a rapid stress 
Table 1. Experimental conditions, materials, dimension of rock cores and numbers of X-ray tomograms acquired in 12 rock deformation experiments.

\begin{tabular}{lcccc}
\hline Rock type & $\begin{array}{c}\text { Experiment } \\
\text { Code }\end{array}$ & $\begin{array}{c}\text { Confining } \\
\text { Stress (MPa) }\end{array}$ & $\begin{array}{c}\text { Sample } \\
\text { Diameter (mm) }\end{array}$ & $\begin{array}{c}\text { \# of X-ray } \\
\text { Tomograms }\end{array}$ \\
\hline Fontainebleau sandstone & FBL01 & 20 & 5 & 184 \\
& FBL02 & 10 & 5 & 47 \\
Mount Etna basalt & ETNA01 & 10 & 4 & 32 \\
& ETNA02 & 10 & 4 & 36 \\
Monzonite & MONZ04 & 35 & 4 & 65 \\
Westerly granite & MONZ05 & 25 & 4 & 80 \\
Green River shale & WG02 & 5 & 4 & 30 \\
& WG04 & 10 & 4 & 66 \\
Anstrude limestone & GRS02 & 20 & 5 & 60 \\
& GRS03 & 20 & 5 & 61 \\
& ANS02 & 20 & 5 & 41 \\
& ANS05 & 5 & 5 & 26 \\
\hline
\end{tabular}

drop that prohibits imaging the rock as it supports decreasing axial stress.

These experiments have been described individually in previous studies from our group, including the experiments on Green River shale (McBeck et al. 2018), Fontainebleau sandstone (Renard et al. 2019a), monzonite (Renard et al. 2017, 2019b), Etna basalt (McBeck et al. 2019) and Anstrude limestone (Renard et al. 2017). The X-ray microtomography data for many of these experiments are available to the community (Renard 2017, 2018a,b,c; Renard \& McBeck 2018)

\subsection{Digital volume correlation}

The code TomoWarp2 (Tudisco et al. 2017) performs the DVC calculations. The DVC method searches for similar patterns of voxels in pairs of 3-D volumes. The parameters of the DVC calculation are constant in each experiment, with 20 voxel node spacing $(0.13 \mathrm{~mm})$ and 10 voxel correlation window size $(65 \mu \mathrm{m})$. The node spacing determines the spatial resolution of the calculation. The correlation window size determines the volume of the cube used to identify similar patterns of voxels. Both of these parameters can influence the magnitude of the strain calculated with DVC (McBeck et al. 2018), so we keep the parameters constant in each experiment.

For each experiment, we perform ten DVC calculations using scans separated by approximately equal increments of cumulative axial strain, producing ten incremental displacement fields between pairs of tomograms (Fig. 1). The DVC calculations track the 3-D displacement field between each scan acquisition, so the displacement vectors reflect the incremental deformation between the stress steps and not the cumulative deformation. Consequently, the incremental strain tensors calculated from these incremental displacement fields quantify the incremental strain between each scan acquisition and not the cumulative strain.

From these displacement fields, we calculate the divergence and curl at each point of the DVC calculation (Fig. S1). Negative and positive values of divergence indicate contractive and dilative strains, respectively. Negative and positive values of curl indicate the left-lateral and right-lateral shear strains, respectively. In order to compare the magnitude of the strain components throughout each experiment, we divide the components by the macroscopic axial strain between each scan used in the DVC calculation, following the procedure of McBeck et al. (2018). This division aids comparison of the magnitude of the strain components at different stress steps in an experiment. Larger increments of imposed macroscopic axial strain will likely produce larger magnitudes of local strain than smaller increments, so dividing the local strains by the magnitude of these increments helps reduce the impact of these varying magnitudes.

\subsection{Experimental rock types}

We focus on the deformation processes throughout 12 experiments on six rock types in this contribution, with two experiments on each rock type. Fontainebleau sandstone is a quartz arenite with a homogeneous mineralogical composition, well-sorted grain size distribution, average grain diameter of $0.25 \mathrm{~m}$ and 3-30 per cent porosity (Bourbie \& Zinszer 1985). Mount Etna basalt is a porphyritic intermediate alkali basalt that experienced quick cooling, producing pores and thermal-induced fractures (Vinciguerra et al. 2005; Benson et al. 2007; Heap et al. 2009). Westerly granite is a crystalline intrusive igneous rock composed of about 40 per cent plagioclase, 25 per cent alkaline feldspar, 29 per cent quartz and minor amounts of biotite, muscovite and chlorite (Rutter \& Neumann 1995). Monzonite is a crystalline intrusive igneous rock, and the monzonite deformed here has a mean grain size of $450 \mu \mathrm{m}$, and contains 18 per cent quartz, 13 per cent biotite, 58 per cent plagioclase, 12 per cent clinopyroxene and minor minerals (Aben et al. 2016). We deformed cores from the organic rich (R-8 unit) Green River shale that includes lacustrine marl/silt sediments that form laminations with 9.9 wt per cent organic matter (Kobchenko et al. 2011). The layering (laminations) of the Green River shale cores were set parallel to the maximum compression direction in these experiments. Anstrude limestone is an oolitic limestone that is nearly monomineralic with 98 per cent calcite (Han et al. 2016). The sandstone, basalt and limestone cores had initial porosities of 5-7 per cent (measured with imbibition and tomography data), 3 per cent (measured with tomography data) and 4-8 per cent (measured with tomography data), respectively. The initial porosities of the other rocks were below 0.5 per cent as calculated with the tomography data.

\section{RESULTS}

\subsection{Local spatial correlation of strain modes throughout loading}

The spatial distribution of strain components with high magnitudes provides insights into the strain accumulation and localization processes within the rocks. Fig. 2 shows the location of high magnitudes 

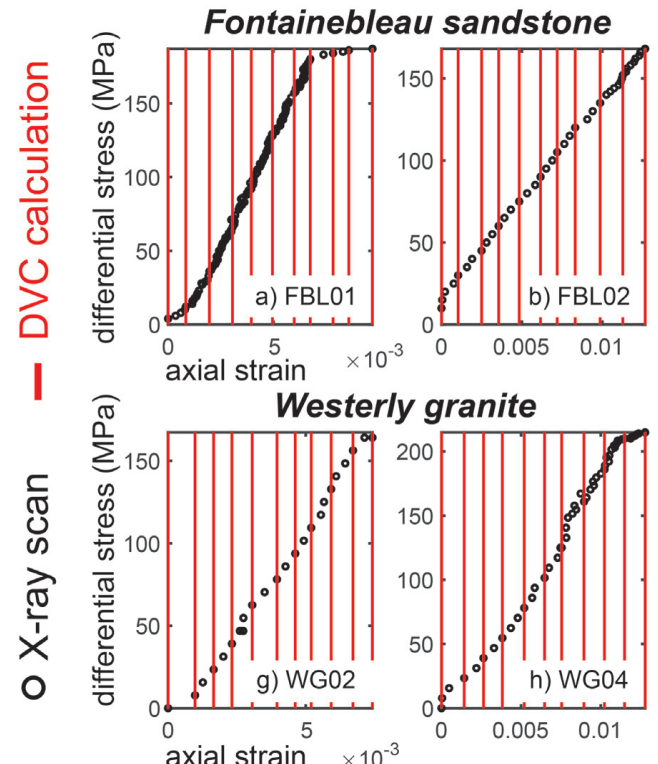

Etna basalt
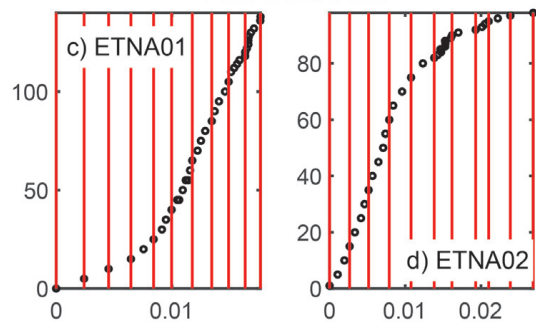

Green River shale
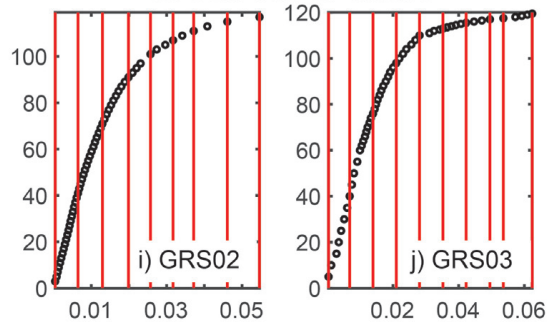

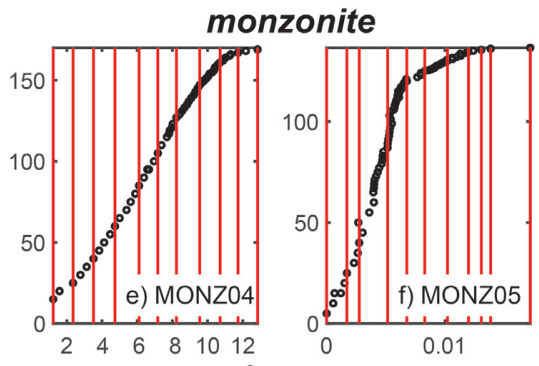

monzonite

Anstrude limestone
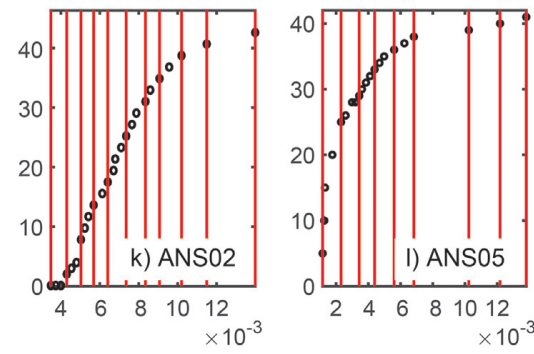

Figure 1. Loading history of experiments on Fontainebleau sandstone (a-b), Mount Etna basalt (c-d), monzonite (e-f), Westerly granite (g-h), Green River shale (i-j) and Anstrude limestone $(\mathrm{k}-\mathrm{l})$. Black dots indicate the axial strain and differential stress conditions when an in situ X-ray tomogram is acquired. Red lines indicate the loading conditions of the tomograms used in the DVC calculations. The pairs of tomograms used in the DVC calculation are separated by approximately constant amounts of macroscopic axial strain in each experiment.
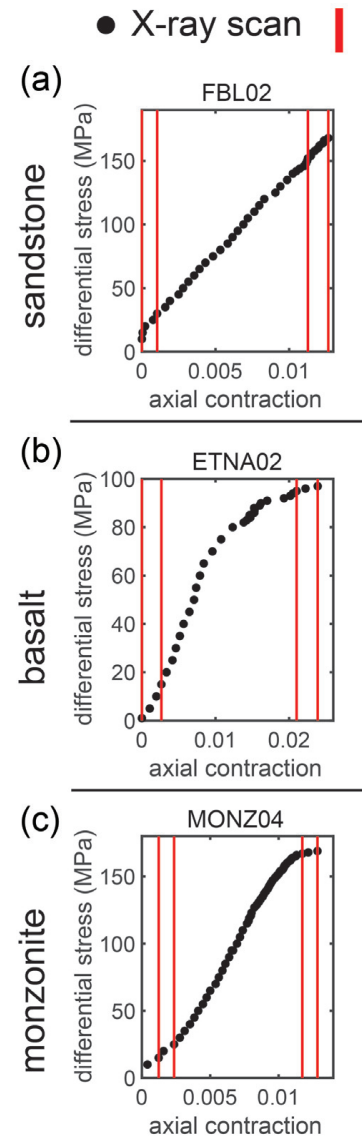

\section{| DVC calculation} low $\sigma_{D}$
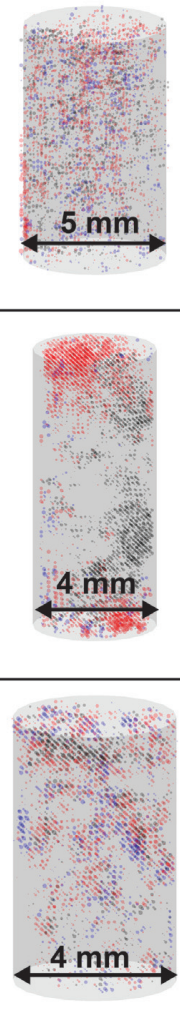

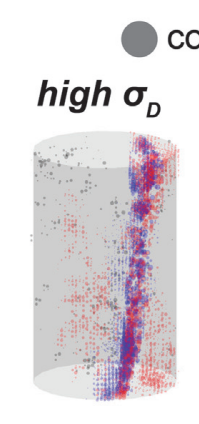

contraction

(d)
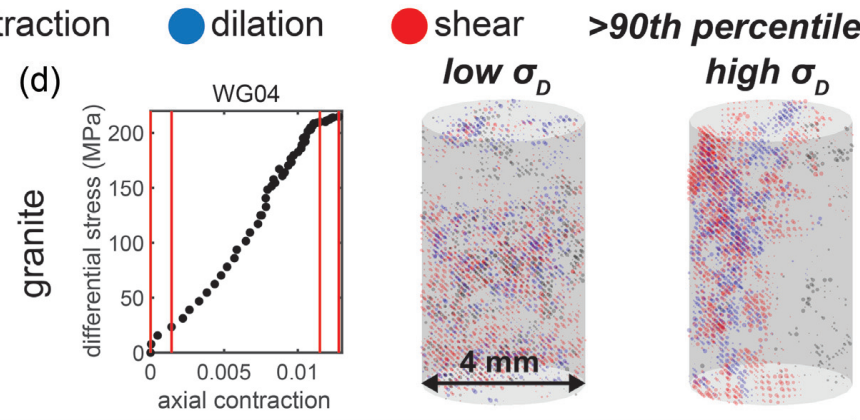

(e)
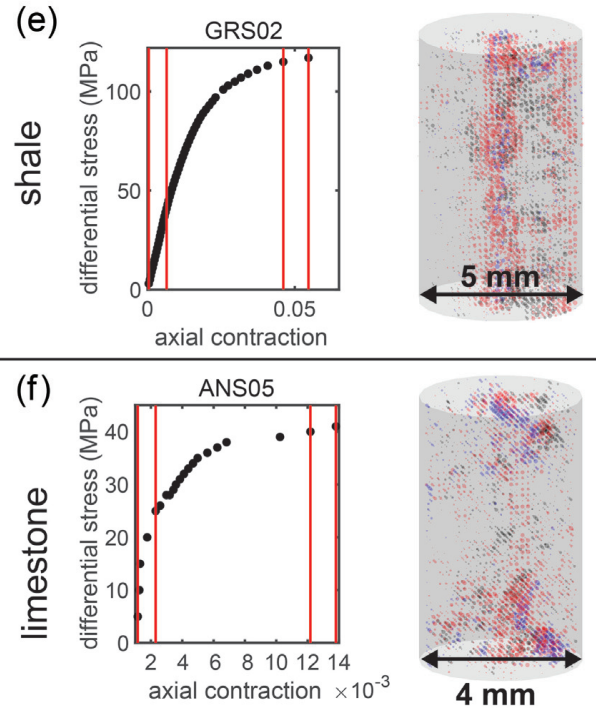

Figure 2. Snapshots of the 3-D strain fields at low and high differential stress, $\sigma_{D}$, for experiments on sandstone (a), basalt (b), monzonite (c), granite (d), shale (e) and limestone (f). The plots show the stress-strain conditions of each acquired tomogram (black dots), and the conditions of the DVC calculations (red lines). Black, blue and red dots in the grey cylindrical cores show where the contraction, dilation and shear strain exceed the 90th percentile of the corresponding strain population, respectively. 
(>90th percentile) of dilation, contraction and shear strain in the first DVC calculation, at the onset of loading under low differential stress, $\sigma_{D}$, and in the final DVC calculation, immediately preceding macroscopic failure and under higher $\sigma_{D}$. In the majority of the experiments, the strain fields are localized close to failure, but the granite and shale experiments do not reveal localization immediately prior to macroscopic failure (Figs $2 \mathrm{~d}$ and e). The strain fields may not show localization because the macroscopic failure of the sample does not include localization and/or the localization occurred at a time interval before or after the final DVC calculation shown here. Segmentation of the scans acquired in the shale and granite experiment reveal that localized through-going fractures detectable at the scan resolution could not be detected preceding the macroscopic failure of the sample (e.g. McBeck et al. 2018). The final DVC calculation includes the scan immediately preceding macroscopic failure, and so this strain field does not include the deformation following macroscopic failure.

Among the experiments that show localization prior to failure, some components of the strain field appear spatially correlated with each other. In the sandstone and basalt experiments, high magnitudes of shear strain develop near high magnitudes of dilation. In contrast, in the monzonite and limestone experiments, high magnitudes of shear strain and dilation develop far from each other in some portions of the core. In the monzonite experiment, localized shear strain develops without accompanying dilation in the final DVC calculation (Fig. 2c). In the limestone experiment, localized dilation develops in the upper portion of the core without localized shear strain (Fig. 2f).

Following these observations, we calculate the Pearson linear correlation coefficient, $\rho$, between the strain components within subvolumes of the DVC calculations throughout each experiment. The Pearson linear correlation coefficient between the populations of two variables, $X$ and $Y$, is calculated from the expectation, $E$, the means of the populations of $X$ and $Y, \mu_{X}$ and $\mu_{Y}$ and the standard deviations of the populations of $X$ and $Y, \sigma_{X}$ and $\sigma_{Y}$, as

$\rho_{X, Y}=\frac{E\left[\left(X-\mu_{X}\right)\left(Y-\mu_{Y}\right)\right]}{\sigma_{X} \sigma_{Y}}$.

Previous work described in Section 2 (e.g. Hamiel et al. 2004b, Stanchits et al. 2006; Tal et al. 2016; McBeck et al. 2019; Renard et al. 2019a,b) suggests that dilation and shear strain should be more strongly correlated than dilation and contraction or shear strain and contraction. This correlation quantifies the spatial relationship between the strain components observed qualitatively in Fig. 2, within subvolumes in the sample (i.e. local spatial correlation). In particular, we compare the mean of each strain component magnitude within each subcube in a 3-D grid separated by $0.2 \mathrm{~mm}$, and thus with $0.2^{3} \mathrm{~mm}^{3}$ volume each, about twice the spatial resolution of the DVC calculation $(0.13 \mathrm{~mm}$, Fig. S1). This procedure produces about 30000 strain values for each calculation of $\rho$ (eq. 1). The correlation coefficients thus track the similarity and differences in strain components within each sampling subcube of $0.2^{3} \mathrm{~mm}^{3}$ volume. We only report the correlation coefficients that are statistically significant, with $p$-values $<0.05$. Positive correlations between strain components indicate that high magnitudes of one component are associated with high magnitudes of the other component. Negative correlations indicate that high magnitudes of one component are associated with low magnitudes of the other component. Values of the correlation coefficient, $|\rho|,<0.3$ indicate weak correlations (or anticorrelations), between 0.3 and 0.5 indicate moderate correlations (or anti-correlations), and $|\rho|>0.5$ indicate strong correlations (or anticorrelations, e.g. Cohen 1988).
To examine the correlation between how each strain component evolves from one DVC calculation to the next, we also calculate the change in the mean of each strain component magnitude at each cube in the sampling grid, and calculate the correlation coefficient between the changes in the means. We do not consider the difference between left- and right-lateral shear strains in this analysis, and perform the correlation calculations using the absolute value of the shear strain. In summary, we report the local spatial correlations between the magnitude of (1) the mean dilation and mean contraction magnitude, (2) the mean dilation and mean shear strain magnitude, (3) the mean contraction and mean shear strain magnitude, (4) the change in the mean dilation and change in the mean contraction magnitude, (5) the change in the mean dilation and change in the mean shear strain magnitude and (6) the change in the mean contraction and change in the mean shear strain magnitude (Fig. 3).

Most of the correlations between the mean of the strain components (Figs $4 \mathrm{a}$ and $\mathrm{b}$ ) are positive in each experiment, except the shale experiment GRS03. This experiment hosts a moderate-strong negative correlation between contraction and dilation, and contraction and shear strain (Figs 4 and S2). In the other experiments, contraction and dilation have moderate-strong positive correlations, and contraction and shear strain have weak-moderate positive correlations. In the shale experiment GRS03, in contrast, increasing contraction does not yield higher magnitudes of dilation or shear strain (Fig. S2).

Whereas the directions (positive or negative) and strengths of the correlations between the pairs of strain component means (Figs 4a and b) are similar in all but one experiment, the correlations between evolving changes of the mean of the strain component (Figs $4 \mathrm{c}$ and d) show greater differences. The correlations between the change in the dilation and contraction, and contraction and shear strain are near zero or negative with weak strength. In contrast, the correlation between the change in contraction and dilation are higher with moderate-strong positive correlations.

These varying signs of the correlation coefficients indicate that the strain accommodation process is dominated by the paired response of changes in dilation and shear strain throughout each experiment, as expected from previous studies (Hamiel et al. 2004b, Stanchits et al. 2006; Tal et al. 2016; McBeck et al. 2019; Renard et al. 2019a, b). Increasing changes in dilation are paired with increasing changes in shear strain. However, increasing changes in contraction are not strongly associated with increasing changes in shear strain or increasing changes in dilation. Whereas the magnitudes of these strain components (Figs 4a and b) are generally positively correlated with each other, the changes of evolving strain components (Figs $4 \mathrm{c}$ and d) are only positively correlated for the paired dilation-shear strain, and not for dilation-contraction and contraction-shear strain.

\subsection{Volumetric frequency of strain accommodation modes}

The observed differences in the correlation coefficients between changes in the mean strain component magnitude prompt a more detailed investigation into the spatial distribution of the modes of strain accommodation. Here, we examine the relative volumes of rock that experience various strain accommodation modes, including the paired increase in dilation and shear strain, the paired increase in contraction and shear strain, and the paired increase in contraction and dilation in individual subvolumes throughout the rock cores (Figs 5). We also report how this partitioning evolves from lower to higher differential stress conditions. We document the relative fraction of rock volume that experience these varying 


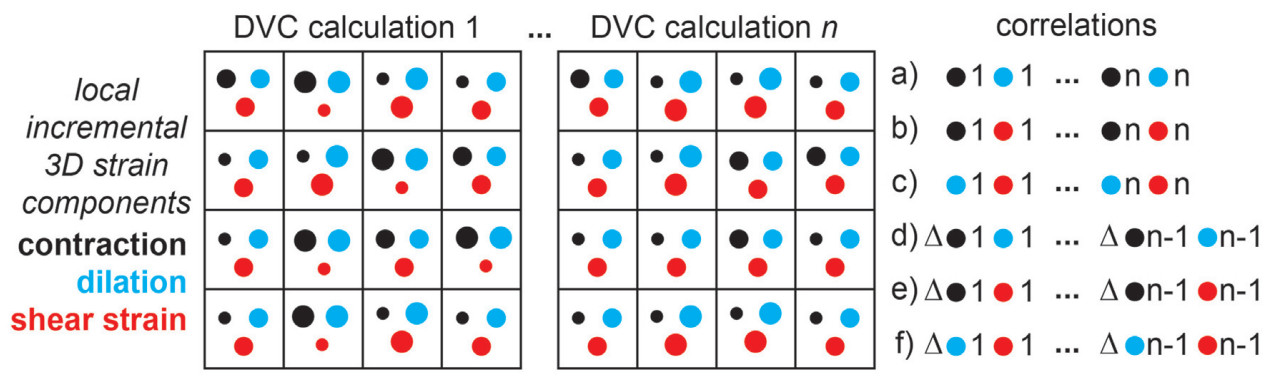

Figure 3. Schematic representation of the suite of correlation calculations. To calculate the correlation coefficient, $\rho$, we calculate the local incremental strain components in subvolumes throughout the rock in $n$ calculations that span the loading of the rock to failure. Then we calculate the mean of each strain component in $0.2^{3} \mathrm{~mm}^{3}$ subvolumes (boxes in figure), about twice the resolution of the DVC calculation. Then we calculate $\rho$ for the pairs of strain components shown in $(\mathrm{a}-\mathrm{f})$, with the black, blue and red dots indicating the contractive, dilatative and shear strains, respectively. The correlations of $(\mathrm{a}-\mathrm{c})$ and $(\mathrm{d}-\mathrm{f})$ use the means of the strain components in each $0.2^{3} \mathrm{~mm}^{3}$ subvolumes, and the change in these means from scan $i$ to $i+1$, respectively.
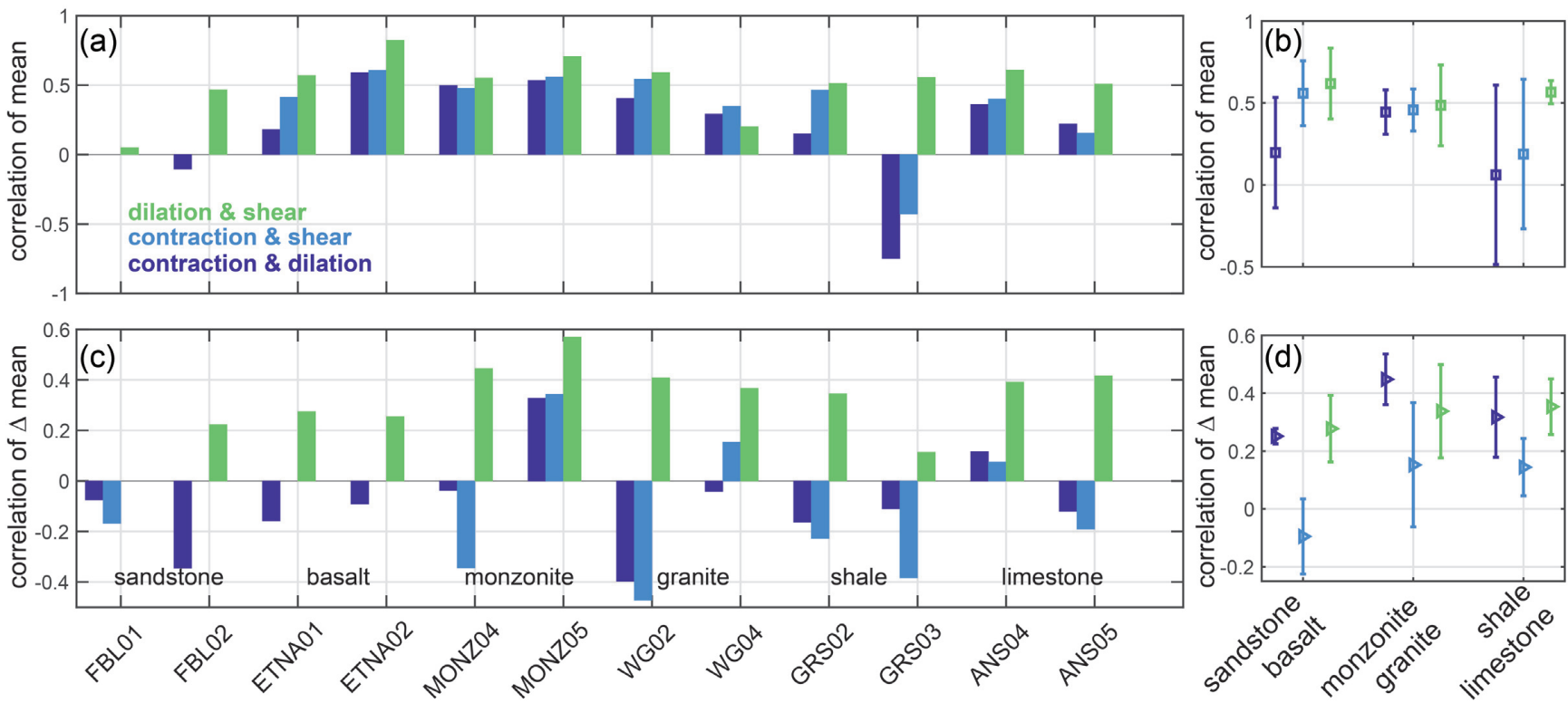

Figure 4. Correlation coefficients between the magnitude of the strain component means (a-b) and change in the strain component means (c-d) at subvolumes throughout each experiment. Correlation between (1) dilation and shear strain, (2) contraction and shear strain, and (3) contraction and dilation shown with (1) green, (2) light blue and (3) dark blue, respectively. (b, d) Mean \pm one standard deviation of the correlation coefficient between the magnitude of the strain means (b) and change in strain means (d) for each of the four experiments on sandstone and basalt, monzonite and granite and shale and limestone.

strain combinations, as measured with the number of cubic subvolumes that experience different strain combinations. These sampling subvolumes are the same as used in the analysis of Section 4.1.

Throughout the full experiment, the most volumetrically frequent mode of local strain accommodation is the paired increase in the mean of dilation and shear strain (within the same subvolume) in all but the granite experiment (WG04) (Figs S3 and 5a). Even in experiment WG04, this mode of strain accommodation is the second most frequent, after the increase in shear strain (Figs S3 and 5). The increase in the mean of only the shear strain is typically the second or third most frequent strain accommodation mode in all experiments (Figs S3 and 5).

The nature of strain accommodation at the first (Fig. 5b) and final (Fig. 5c) experimental stages captured in the DVC calculation shows that the most frequent mode of strain accommodation evolves from lower to higher differential stress conditions. Early in loading, the paired increase in contraction and dilation is generally one of the most frequent modes. This mode of strain accommodation is the most frequent in the experiments on basalt, monzonite and granite, and the second most frequent in the experiments on sandstone and shale in the first DVC calculation. In the limestone experiments, the most frequent mode in this early stage is the increase in only the shear strain.

Immediately preceding macroscopic failure, the paired increase in dilation and shear strain is one of the most frequent modes, as well as the increase in only shear strain (Fig. 5c). The paired increase in dilation and shear strain is the most frequent mode in the basalt, monzonite, granite and shale experiments at this stage. This mode is the second most frequent in the sandstone and limestone experiments preceding failure. The most frequent mode in the sandstone and limestone experiments is the lone increase in shear strain, and the decrease in all of the strain components, respectively.

\subsection{Comparing strain accumulation and localization}

The previous analyses link the spatial coincidence of different strain components, and compare the evolution of this coincidence 

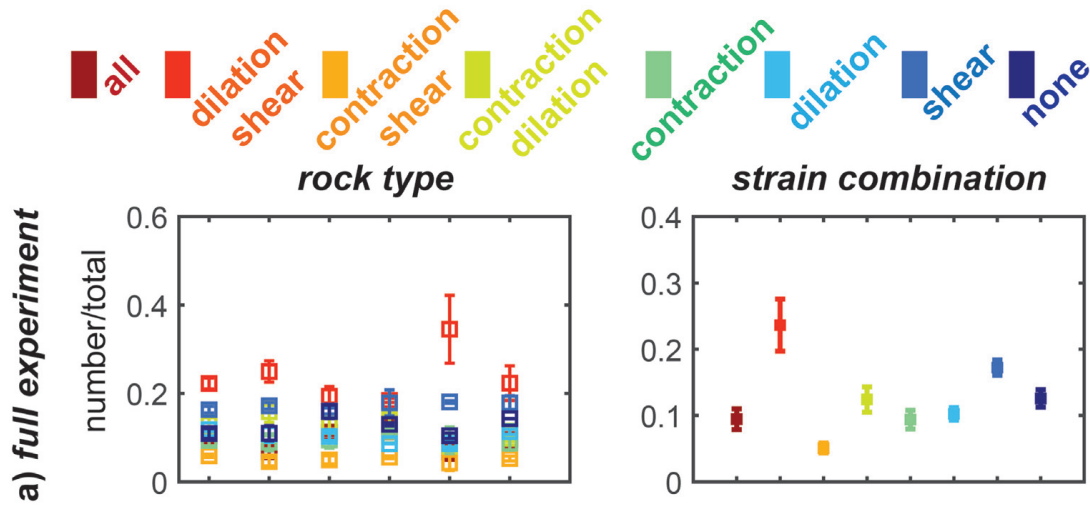

strain combination
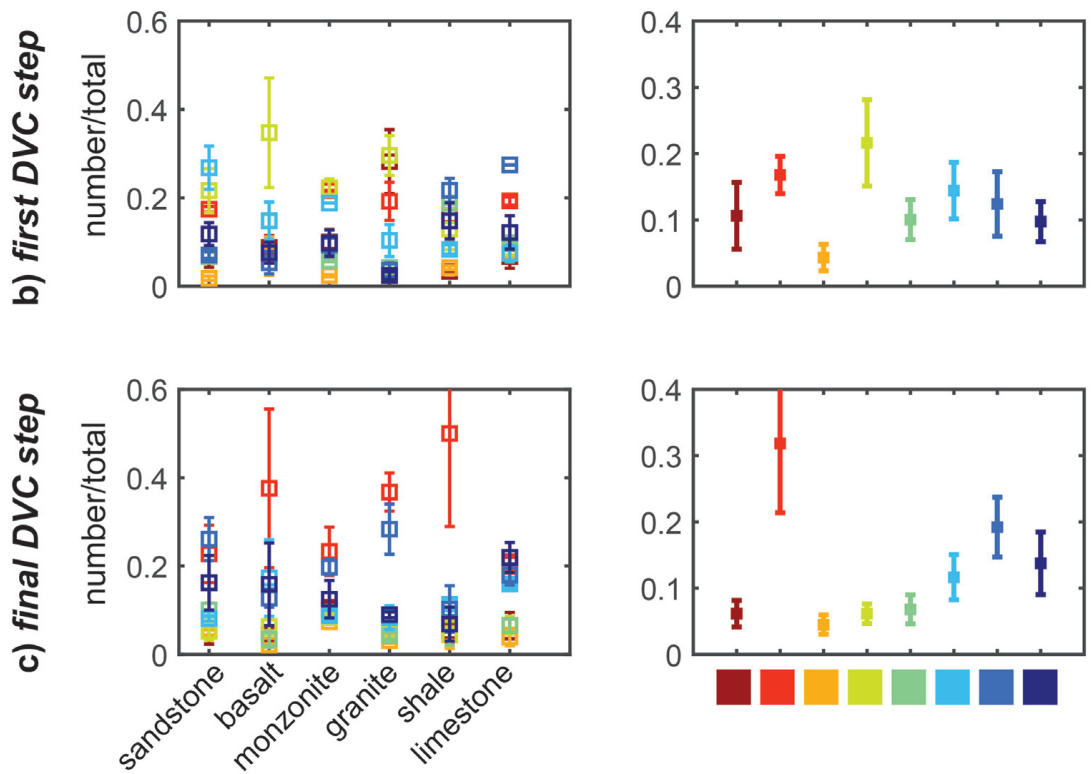

Figure 5. Summary of the differences in strain accommodation modes at different stages of loading: (a) throughout the full experiment, (b) first DVC calculation and (c) final DVC calculation. Left-hand column: mean \pm one standard deviation of the normalized strain accommodation frequency, $n / t$, of the two experiments of each rock type. $n / t$ is measured as the number of voxels occupied by the increase in none, all, one, or two of the strain components, $n$, out of the total number of voxels, $t$. Right-hand column: mean \pm one standard deviation of $n / t$ for all the experiments.

from lower and higher differential stress conditions. The differences in this evolution with differential stress prompt an examination of the strain accommodation mode throughout each experiment with a higher temporal resolution. In particular, we track the mean amplitude and rock volume occupied by four strain components: dilation, contraction, left-lateral shear strain and rightlateral shear strain at each DVC calculation (Figs S4 and 6). Previous work indicates that the evolution of the rock volume occupied by dilation relative to contraction, and the volume occupied by left-lateral relative to right-lateral shear strain, signal approaching macroscopic failure (McBeck et al. 2019; Renard et al. 2019b).

We adopt the convention of showing when the dilation exceeds the contraction, and when the left-lateral shear strain exceeds rightlateral shear strain (Fig. S4, Figs 6 and 7). Because the mean and volume of the dilation tend to increase, while those properties of the contraction tend to decrease with loading, we mark the axial strain range when the dilation exceeds the contraction. The axial strain range when the left-lateral shear strain exceeds the rightlateral shear strain is no more significant than when the right-lateral shear strain exceeds the left-lateral shear strain in our experiments. Marking the intervals when right-lateral shear strain exceeds the left-lateral shear strain would produce the same patterns as found with the adopted convention.

In all but one of the limestone experiments (ANS02), the mean of the dilation exceeds the mean of the contraction at some stage (Fig. S4, Figs 6 and 7). Similarly, in all but this experiment, the volume of rock occupied by dilation exceeds that occupied by contraction at some stage. Some of the experiments also show that the mean or volume of one of the shear strain components exceeds the other for intervals of axial strain. In some experiments (i.e. FBL01, GRS02 and WG02) the axial strain interval over which the mean of the dilation exceeds the mean of the contraction aligned with the axial strain interval over which the volume occupied by the dilation exceeds the volume occupied by the contraction (Figs 6a, $d$ and e). In fewer experiments, the intervals over which both the mean and volume of left-lateral shear strain exceeds the right-lateral shear strain coincide (i.e. FBL01, Fig. 6a).

Fig. 7 shows the experiment stages (macroscopic axial strain) over which the dilation exceeds the contraction, and the left-lateral 

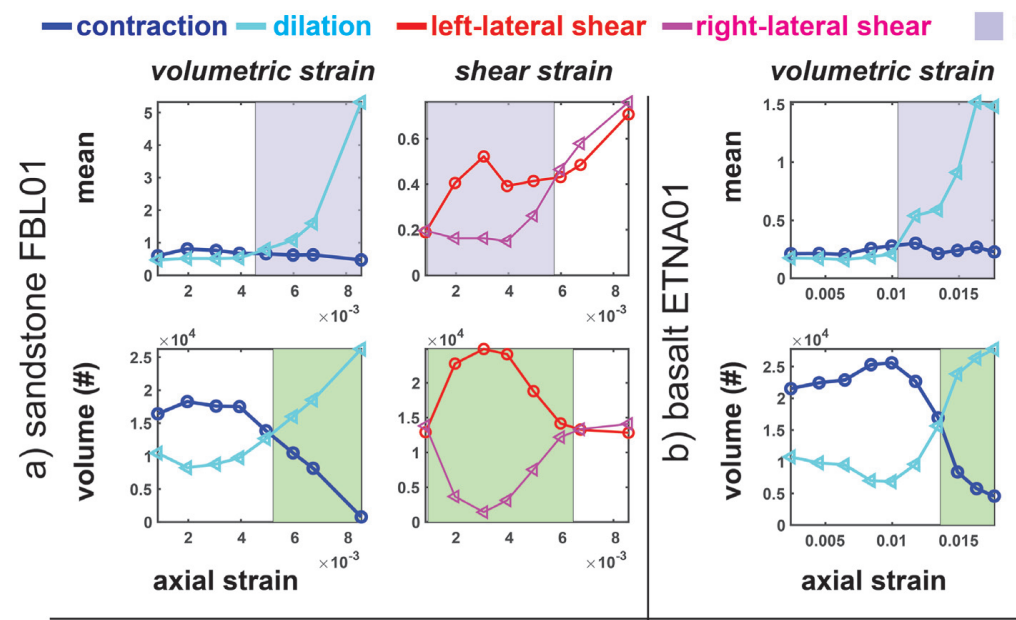

mean volume
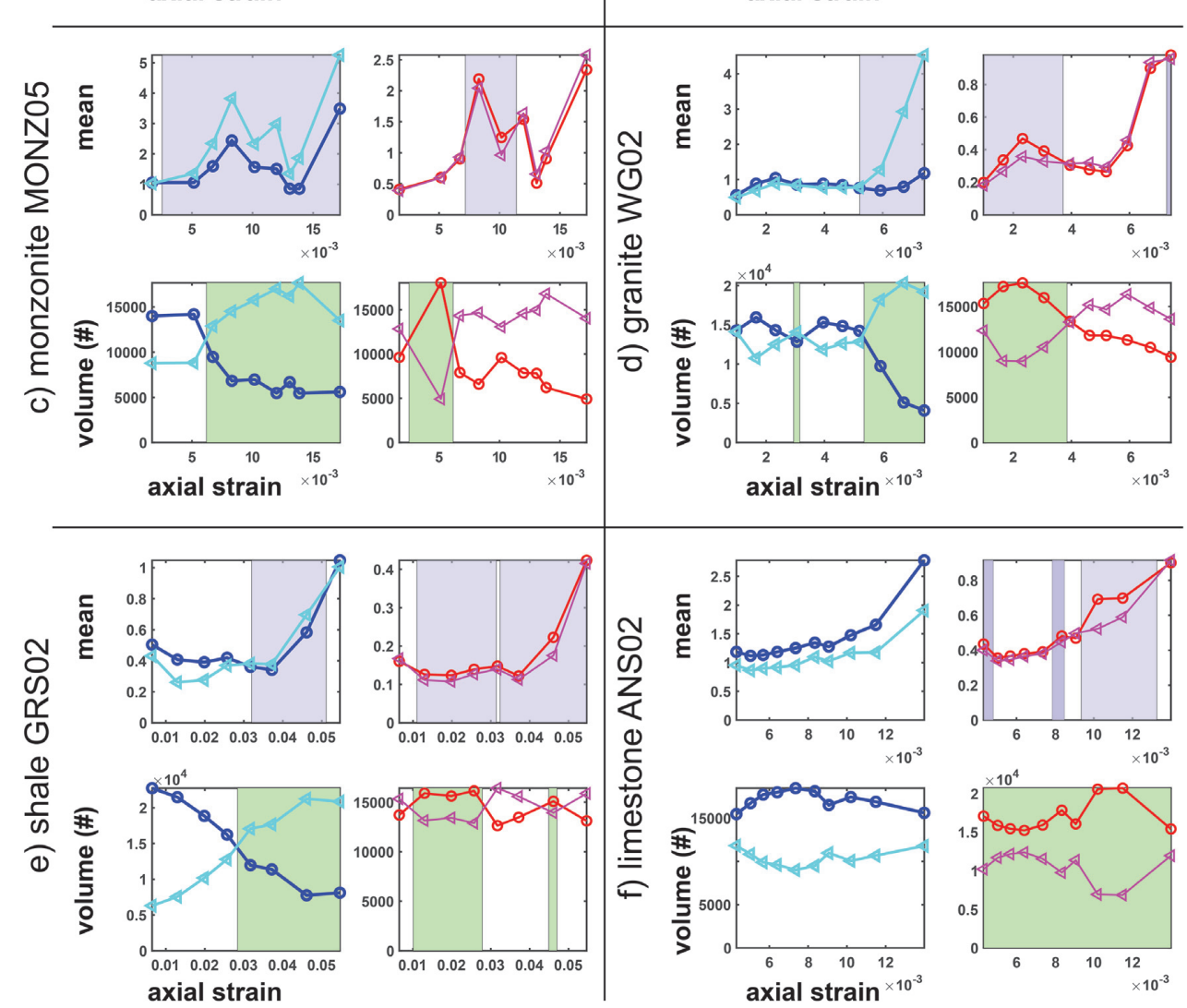

Figure 6. Evolution of strain accommodation throughout time (macroscopic axial strain) in six experiments on (a) sandstone, (b) basalt, (c) monzonite, (d) granite, (e) shale and (f) limestone. First row of each subsection shows the mean of the strain components, including the dilation, contraction, left-lateral and right-lateral shear strain. Second row shows the volume of the rock that each strain component occupied as the number of voxels. Left and right columns of each subsection show the dilation (light blue) and contraction (dark blue), and left-lateral (red) and right-lateral (pink) shear strain, respectively. Blue and green rectangles highlight the time when the dilation mean (blue) or volume (green) exceeds the contraction mean or volume, and when the left-lateral shear strain mean or volume exceeds the right-lateral shear strain mean or volume, respectively. Fig. S4 shows these data for the other six experiments.

shear strain exceeds the right-lateral shear strain, in terms of either the mean magnitude or volume. In all but one of the experiments (ANS02), the normalized axial strain when the dilation first exceeds the contraction occurred at $0.3-0.7$ axial strain from failure (Fig. 7). In the limestone experiment $\mathrm{ANS02}$, the volume and mean of the contraction exceed those properties of the dilation throughout the full experiment, consistent with the expected pore-collapse mechanism operating in limestone during brittle failure (e.g. Zhu et al. 2010). In all of the experiments, the time when the mean dilation exceeds the mean contraction overlaps the time when the volume occupied by dilation exceeds the volume occupied by contraction. When higher magnitudes of dilation permeate the rock, the dilative strains also occupy more volume than the contractive strains.

For most experiments (e.g. FBL02, MONZ05 and GRS03), the timing of the shear strain accommodation modes is not coincident. In these experiments, the mean of the left-lateral shear strain exceeds the mean of the right-lateral shear strain throughout a time 


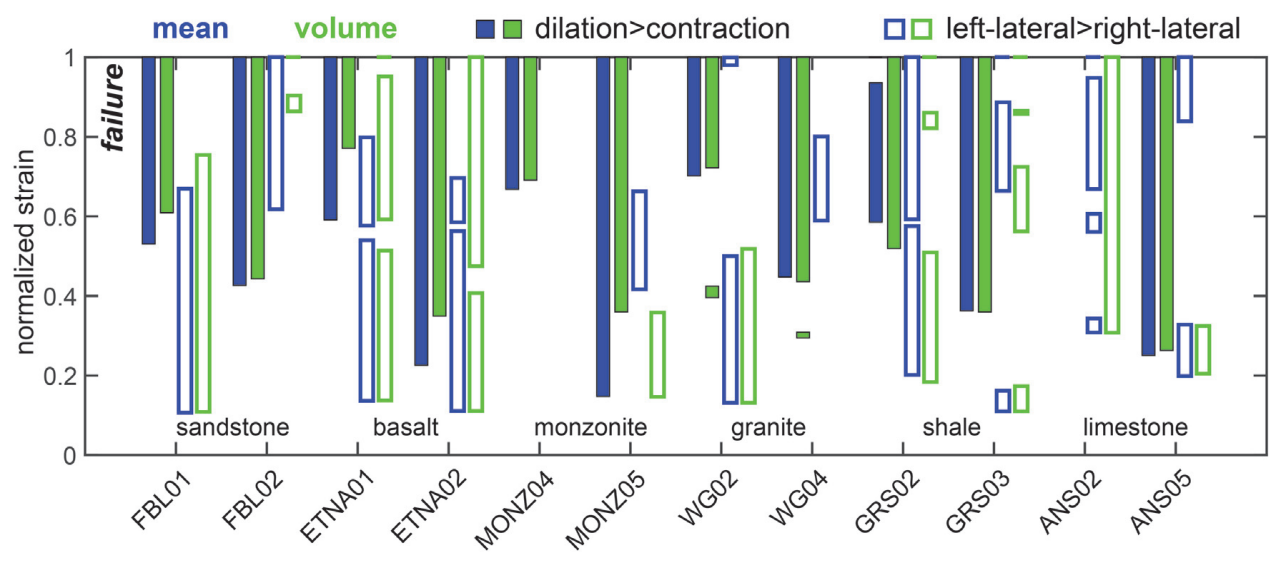

Figure 7. Time periods during each experiment when the mean or volume of the dilation exceeds the contraction, and when the mean or volume of the left-lateral shear strain exceeds the right-lateral shear strain. Time is reported as the normalized strain: the axial strain divided by the axial strain immediately preceding macroscopic failure, with zero at the onset of loading and one at failure. Time periods that correspond to the mean and volume of the strain components shown with blue and green, respectively. Time periods that correspond to the volumetric strains and shear strains shown with filled rectangles and outlines of rectangles, respectively. The mean and volume of the dilation exceeds the contraction by the end of the experiment in 11 of 12 experiments. Near the axial strain when the dilation mean exceeds the contraction mean, the dilation volume also exceeds the contraction volume. This synchronicity of the mean and volume identified for the volumetric strains does not occur for the shear strain components. Dilation tends to delocalize while gaining strength (increasing in mean and volume at the same time), whereas shear strain tends to localize or remain at similar volumes while gaining strength (increasing in mean, but not in volume, or vice versa).

interval when the volume occupied by the left-lateral shear strain does not exceed the volume occupied by the right-lateral shear strain. Higher magnitudes of shear strain are not always (or often) coincident with permeating larger volumes. The few experiments in which larger volumes of left-lateral shear strain occur at the same axial strain ranges of larger means of left-lateral shear strain include the sandstone experiment FBL01 and both basalt experiments.

To more directly compare the synchronicity of the strain accumulation and localization processes, we report the percentage of the macroscopic axial strain (i.e. experimental time) that both the mean and volume of one of the strain components (dilation or left-lateral shear strain) exceed the other component (contraction or right-lateral shear strain, Fig. 8). We report this percentage out of the total axial strain corresponding to the mean magnitude or fractional volume, whichever is longer (e.g. Fig. 8a). In only one experiment (sandstone, FBL01), the percentage of overlap time when both the mean and volume of the left-lateral shear strain exceed the right-lateral shear strain is higher than the percentage of overlap time for the volumetric strains (Fig. 8c). In seven experiments, the percentage of overlap time of the volumetric strains is higher than the overlap time of the shear strains. In three experiments, the overlap times of the volumetric and shear strains are similar. Grouping the overlap times by rock types (Fig. 8b), and calculating the average of the overlap time of each pair of experiments on the same rock type, reveals that only the pair of experiments on sandstone have average overlap times of the shear strain that exceed the average overlap times of the volumetric strains. For all the other rock types, the average overlap times of the volumetric strains are higher than the average overlap times of the shear strains.

Increases in the magnitude of dilation generally are coincident in time with increases in the volume experiencing this dilation. In contrast, increases in the magnitude of shear strain are coincident with increases in the volume rock experiencing the strain with lower frequency than the volumetric strains. Whereas dilation tends to occupy larger volumes (delocalizing) as it gains strength throughout each experiment, shear strain tends to occupy the same or smaller volumes (localizing) as it gains strength.

\subsection{Tracking the interaction distance of strain modes}

Building on the previous observations, we now examine how the evolving local volumetric and shear strains influence the strain network within the entire rock sample throughout loading. We calculate the Pearson correlation coefficient between the same strain components at pairs of subvolumes in the core with increasing distances at each differential stress step. The distribution of correlation coefficients at each stress step evolves from one at zero distance (when the same subvolumes are correlated to each other) to near zero at distances close to the diameter of the rock core (4 mm, Fig. S5). The correlation length is taken from the distribution of correlation coefficients as the distance at which the correlation coefficient decreases below 0.5 . We track the correlation length in each experiment for the strain components of contraction, dilation, shear strain (including both the left- and right-lateral shear), and the separate left- and right-lateral shear strains. Fig. S5 shows the distribution of correlation coefficients with distance for half of the experiments, with one example for each rock type, and the correlation lengths derived from these distributions of the five strain components. Fig. 9 shows how the correlation lengths evolve with time (axial strain) for the set of experiments shown in Fig. S5.

In some experiments, such as for sandstone and granite, the correlation lengths of the dilation, shear strain, and left-and right-lateral shear strains increase towards failure (Figs 9a and d). In other experiments, such as for basalt, these correlation lengths decrease towards failure (Fig. 9b). In the monzonite experiment, the correlation lengths of each strain component increase somewhat throughout the loading (Fig. 9c). The evolving correlation lengths of the shale and limestone samples do not show a systematic trend with loading. In these experiments, the correlation lengths of different strain components at a given stress step differ from each other by $0.5-2 \mathrm{~mm}$. 

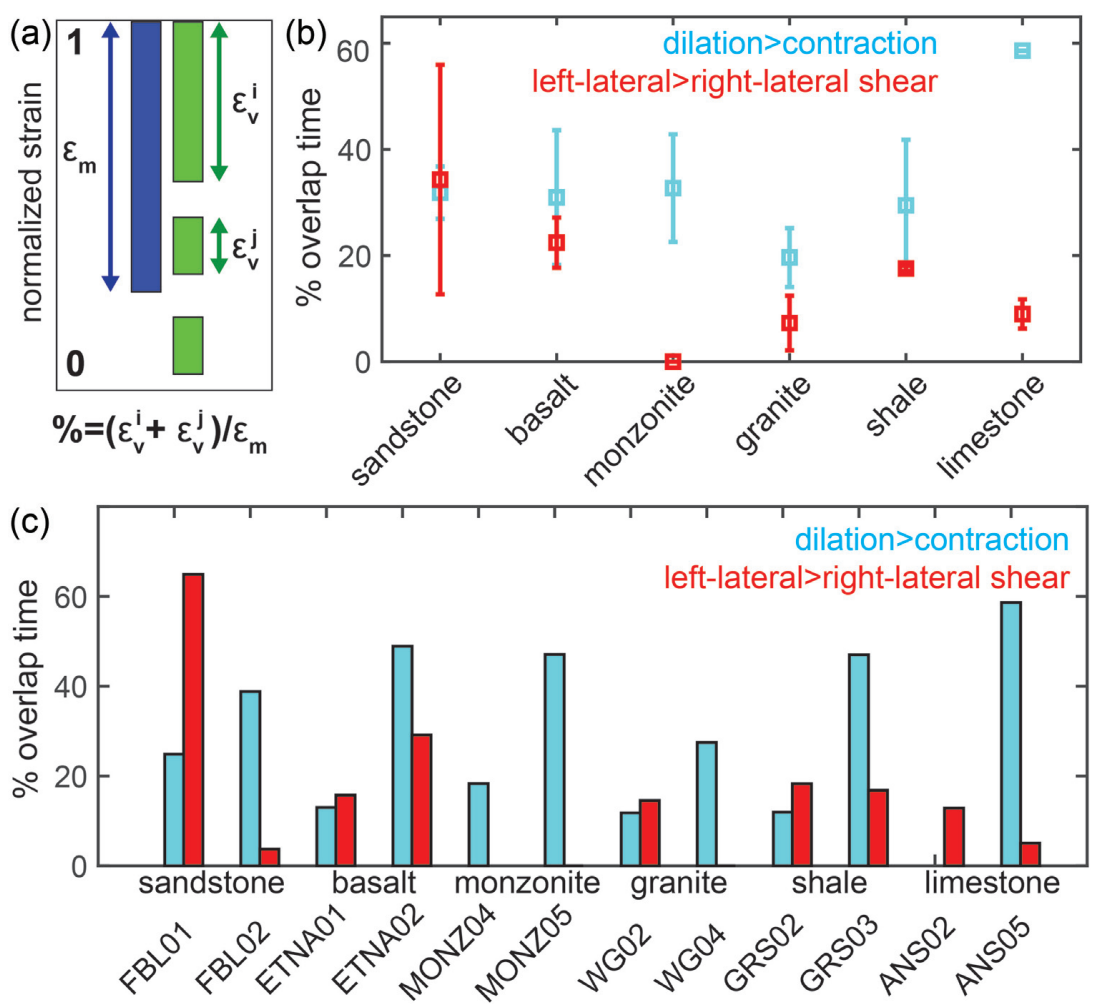

Figure 8. Percent of overlap time when both the mean and volume of a strain component (dilation or left-lateral shear strain) exceeds the other strain component (contraction or right-lateral shear strain). (a) Sketch of quantities used to calculate the per cent overlap time, using the range of normalized axial strain when the mean, $\varepsilon_{m}$, and volume, $\varepsilon_{v}$, of a strain component exceeds the other strain component. Percent overlap time for rock types as the mean \pm one standard deviation of each pair of experiments (b), and for individual experiments (c) when the dilation exceeds the contraction (blue) and left-lateral shear strain exceeds the right-lateral shear strain (red). The per cent overlap time of the volumetric strains tends to be larger than the per cent overlap time of the shear strains.

In contrast, in the other experiments, the correlation lengths of the dilation and shear strain components are more similar at a given stress step.

To compare the evolution of the correlation lengths across all 12 experiments, we show the correlation length at the first and final DVC calculation of each experiment, and the difference in the lengths between these times (Fig. 10). In the first DVC calculation, under lower differential stress, the dilation tends to produce the lowest correlation length of the strain components for each rock type (Figs 10a and b). At this stage, the correlation lengths of the contraction and shear strains tend to be similar to each other, and higher than the dilation. In the final DVC calculation, under higher differential stress, the correlation length of the contraction tends to be lower than the other strain components (Figs 10c and d). The correlation length of the dilation tends to be larger than the contraction, and equal or greater than the length of the shear strains. The difference in the correlation length between the first and final DVC calculations shows that the dilation generally increases by the largest lengths throughout loading (Figs 10e and f). In contrast, the contraction generally decreases in length with loading, or remains at a similar value. The correlation length of the contraction tends to decrease with loading for the sandstone, basalt, monzonite and granite experiments, but stays at a similar length for the shale and limestone experiments (Fig. 10f). The change in the correlation length with loading for the shear strain, and the individual left- and right-lateral shear strains is on average near zero for the groups of rock types, but with ranges above and below zero by up to $1 \mathrm{~mm}$ for each individual experiment. An analysis that accounts for correlations in different directions, such as parallel and normal to the ultimate final failure zone, may provide additional useful information, but is left for future work.

Tracking the change in the correlation length through time suggests that the dilatational strain evolves from influencing the smallest volume of rock of the strain components under lower differential stress to the largest volume of rock under higher differential stress. This increasing interaction distance of the dilatational strain, and generally more limited interaction distance of the shear strains, agrees with our observation that dilation tends to occupy larger rock volumes when increasing in magnitude, whereas the shear strain occupies smaller volumes (i.e. localizes) when increasing in magnitude (e.g. Figs 7 and 8).

\section{DISCUSSION}

\subsection{Spatial coincidence of strain modes}

Digital volume correlation of tomography data acquired in 12 triaxial deformation experiments on six rock types reveal the interplay of local contraction, dilation and shear strain when approaching macroscopic failure. Our work indicates that the strain partitioning process in crustal rocks under the triaxial conditions of the upper crust is dominated by the synchronous expression of multiple strain modes (Figs 4, 5 and 11a). The strengths of local correlations between each pair of strain components in the examined subvolumes are similar to each other (Fig. 4). The link between shear strain and dilation has been well-recognized in the brittle failure of rocks under triaxial compression (e.g. Hamiel et al. 2004b, 

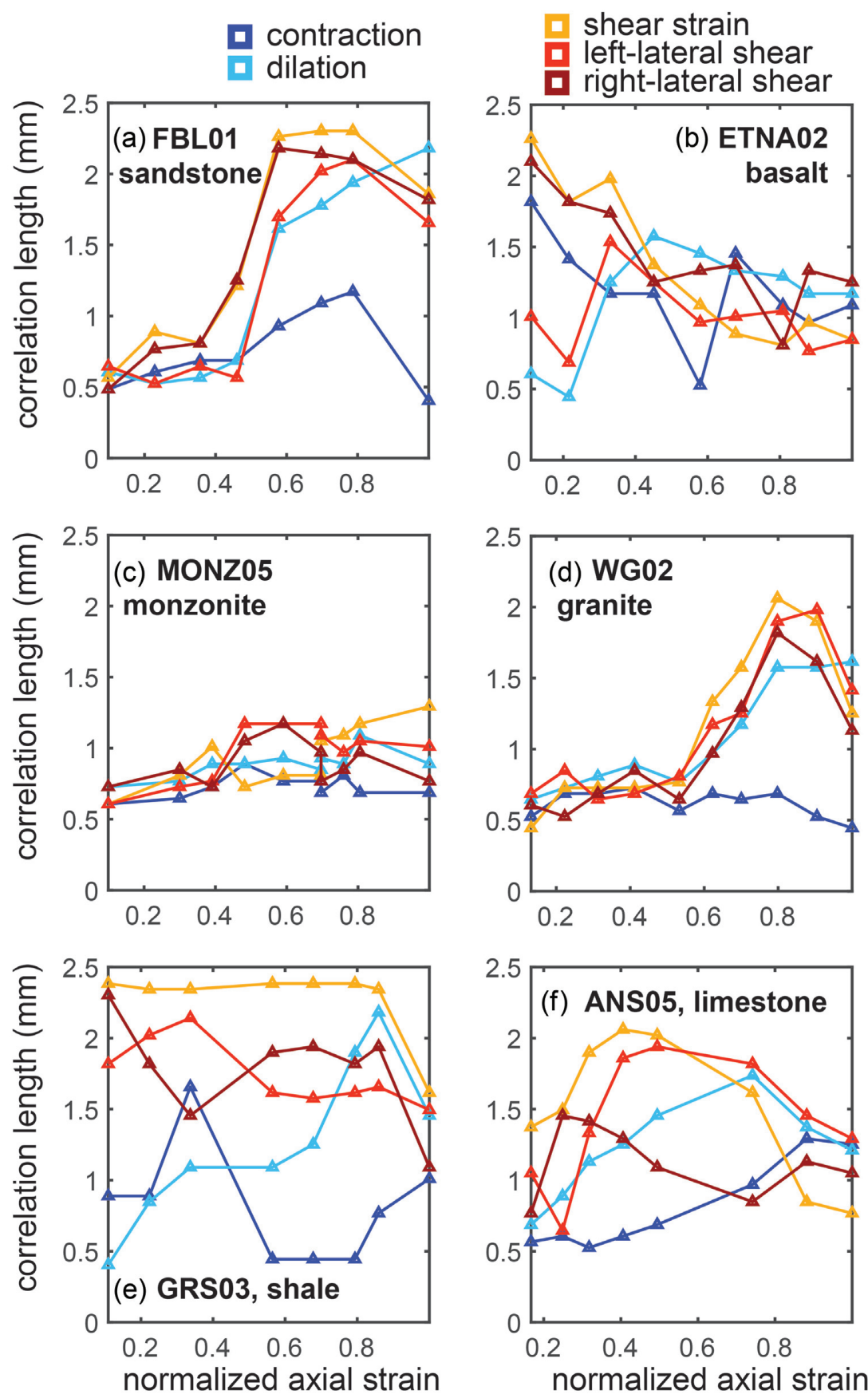

Figure 9. Correlation length of each strain component throughout loading in six example experiments. Fig. S5 shows the distribution of the correlation coefficient with distance for each of these experiments, and the correlation length. In some experiments, the correlation length increases with loading, while in other experiments the correlation length decreases or remains similar.

Stanchits et al. 2006; Tal et al. 2016; McBeck et al. 2019; Renard et al. 2019a,b), and shear strain associated with contraction has been recognized in fewer geologic systems such as cataclastic compaction band development (e.g. Aydin \& Johnson 1983). However, the co-existence of contraction and dilation in small subvolumes has received less attention (e.g. Renard et al. 2019b). The similarity of the strengths of the correlations may occur because we track this correlation throughout triaxial loading, with increasing differential stress. The similar positive correlation strengths suggest that the different strain modes play complementary roles during deformation. Loading accelerates the strain accumulation process, producing a synchronous increase in the magnitude of local incremental contraction, dilation and shear strain.

The correlations in the changes of the strain components align with previous inferences about the link between dilation and shear strain, relative to contraction and shear strain (Fig. 4). In particular, the strength of the correlation between the change in dilation and shear strain is higher than the correlation between the change in contraction and shear strain in all of the experiments. Discrete compaction bands do not develop in any of the experiments, so the link between contraction and shear strain is expected to be weak. When rough surfaces slip, they must either abrade existing asperities or 


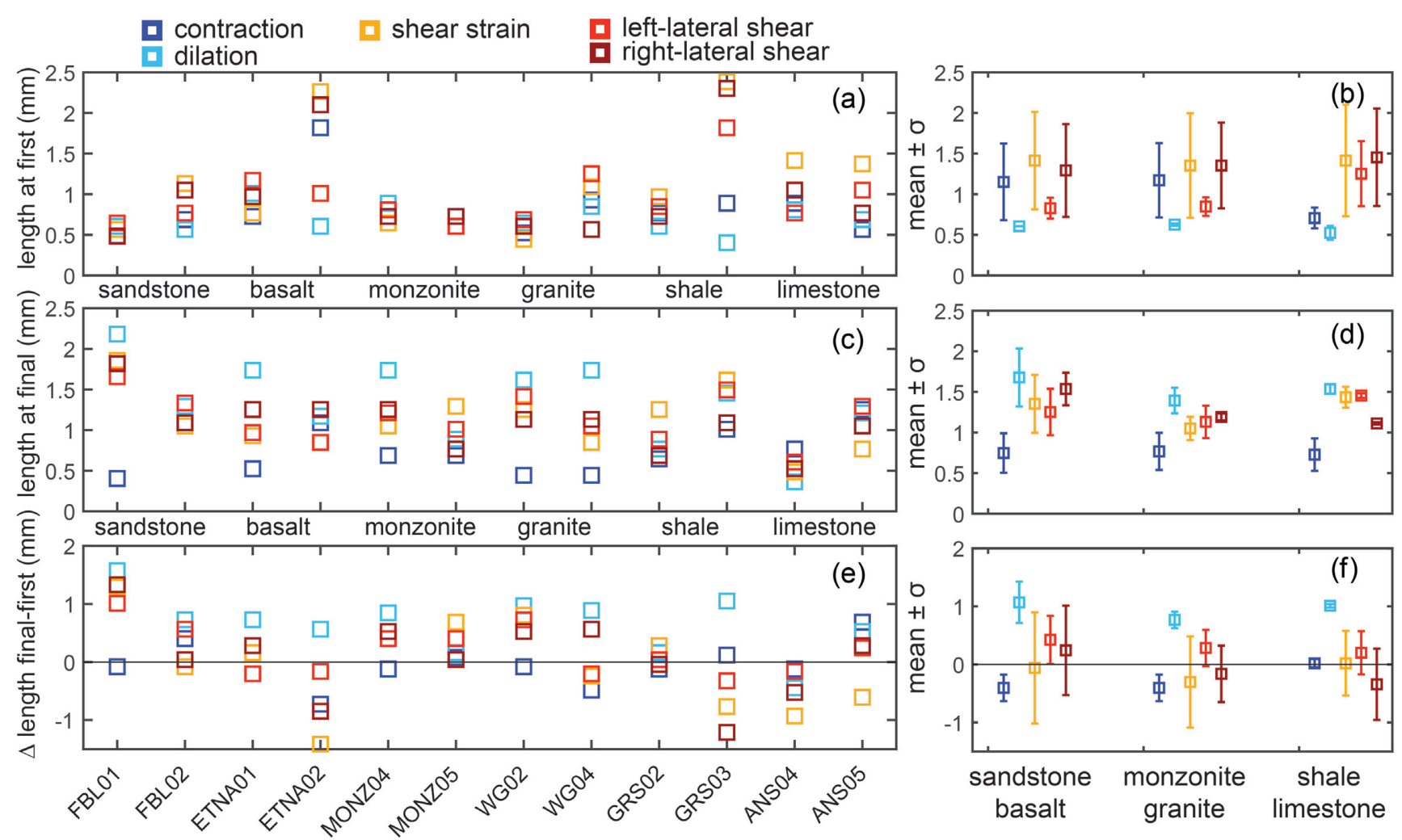

Figure 10. Evolution of the correlation length of strain components in each experiment (a, c, e) and as averages of groups of experiments on similar rock types $(b, d, f)$. Correlation length at the first (a, b) and final (c, d) DVC calculations. (e, f) Difference in the lengths between the first and final DVC calculations. In the first calculation, under lower differential stress $(a, b)$, the dilation tends to have the lowest correlation length of the strain components. Under higher differential stress (c, d), the dilation tends to have the highest correlation length of the strain components. Consequently, the dilation correlation length tends to increase by the largest magnitudes $(\mathrm{e}, \mathrm{f})$. These trends are consistent among the six rock types, and groups of rock types.

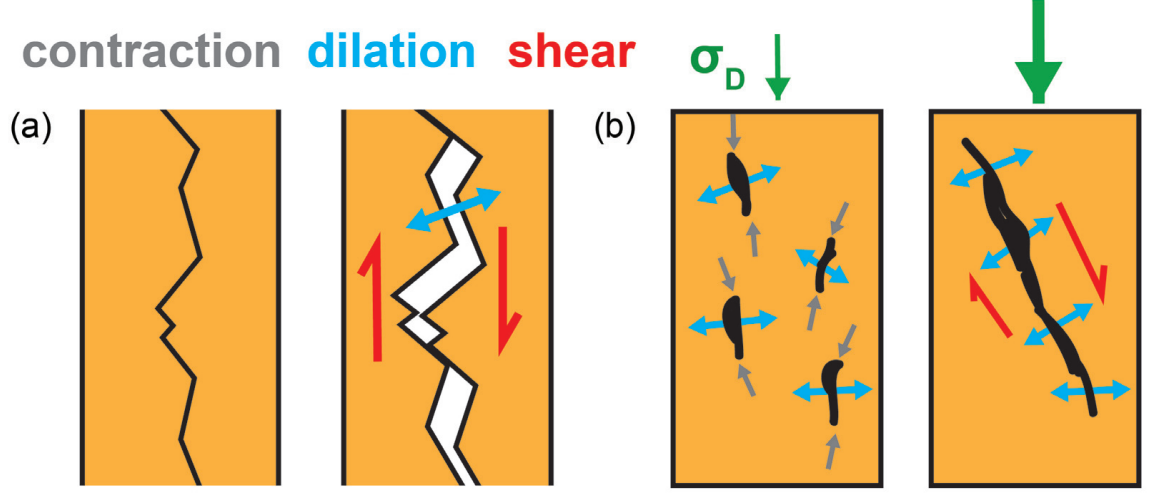

(c)

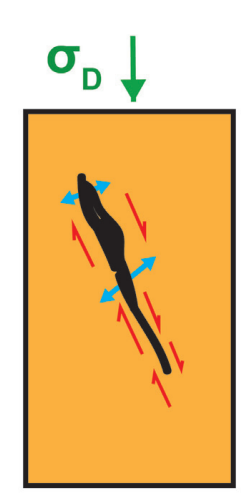

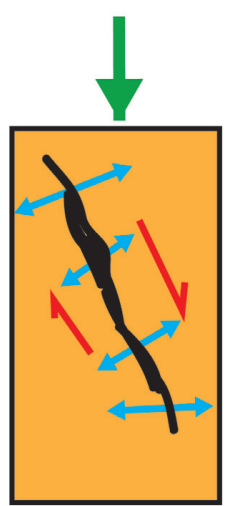

Figure 11. Schematic representation of the strain accumulation and localization process. (a) To accommodate shear strain (red arrows) along rough fractures, portions of the fracture surfaces must dilate (blue arrows). (b) Under lower differential stress (left-hand panel), the paired increase in contraction and dilation dominates deformation. (b) Under higher differential stress (right-hand panel), the paired increase in dilation and shear strain dominates deformation. (c) The diverging behaviour of the strain localization and accumulation process of volumetric and shear strains. Increases in the magnitude of dilation (shown with the thickness of blue arrows) are paired with increases in the volume of rock experiencing dilation (shown with the number of blue arrows). In contrast, increases in the magnitude of shear strain (shown with the thickness of red arrows) are not often paired with increases in the volume of rock experiencing shear strain (shown with the number of red arrows). The correlation length of dilation tends to increase from lower to higher differential stress. In contrast, the correlation length of shear strain does not systematically increase or decrease across all 12 experiments.

dilate, producing the strong link between the change in dilation and shear strain (e.g. Figs 4 and 11a).

The most volumetrically frequent mode of strain accommodation throughout each complete experiment is the paired increase in dilation and shear strain, and not the individual increase in one of the strain modes, in 11 of 12 experiments (Fig. 5). Under relatively low differential stress, the most common strain accommodation mode is the paired increase in dilation and contraction (Figs 5 and 11b). The DVC analysis suggests that as preexisting pores close due to increased axial and confining stress, dilation occurs in regions near 
the contracting volumes (Fig. 11b). The results agree with previous qualitative observations of the spatial relationship between high magnitudes of dilation and contraction recognized from DVC of Xray tomography scans of monzonite (Renard et al. 2019b). Earlier work has noted the tendency of porous rocks to macroscopically contract at the onset of loading (e.g. Brace et al. 1966). First motion polarity analysis of acoustic emissions in experiments on basalt and granite indicate that collapse-type events dominate deformation under hydrostatic loading (Stanchits et al. 2006). Alternating increases and decreases in gravity between flank eruptions on Mount Etna may have arisen from the opening of fractures and local contraction, and not only from magma movement prior to failure (e.g. Carbone et al. 2014).

Under higher differential stress, immediately preceding macroscopic failure, the most volumetrically common strain accommodation mode is the paired increase in dilation and shear strain (Figs 5, 11b). The results indicate that the dilation and shear strain magnitudes increase from one DVC calculation to the next by similar magnitudes within each subvolume (Fig. 4), and that this paired strain combination occupies the largest volume within the rock out of all the possible strain combinations (Fig. 5). Previous work has identified the importance of both dilation and shear deformation in rock failure (e.g. Stanchits et al. 2006). This may arise from micromechanical processes that include (1) the opening of smaller modeI fractures that link into through-going faults that accommodate shear strain detectable at the macroscopic scale (e.g. Petit \& Barquins 1988; Moore \& Lockner 1995), (2) the propagation of tensile fractures aligned parallel to $\sigma_{1}$ that form columns of rock that rotate to allow macroscopic shear (e.g. Peng \& Johnson 1972), and/or (3) the opening of tensile fractures aligned parallel to $\sigma_{1}$ at the tips of wing-cracks inclined relative to $\sigma_{1}$ that accommodate slip (e.g. Wong and Chau 1998). The spatial resolution of the DVC analyses presented here does not enable distinguishing between these different micromechanisms, but does indicate that these processes are consistent with the high volumetric frequency and strong positive correlation of the coeval development of dilation and shear strain.

The difference in the most volumetrically frequent strain accommodation mode from lower (contraction and dilation) to higher (dilation and shear strain) differential stresses suggests that different failure criteria may apply under these different stress conditions. Under lower differential stresses, criteria that describe the conditions of pore-collapse and tensile failure (e.g. Wong et al. 1997) may be the most successful at predicting local failure. Under higher differential stresses, criteria that incorporate micromechanisms that accommodate both dilation and shear (e.g. Peng \& Johnson 1972; Petit \& Barquins 1988; Moore \& Lockner 1995; Wong \& Chau 1998) may be the most successful for predicting progressive development of local processes that produce macroscopic failure. When considering the complete loading history of an experiment under triaxial compression, or progressively increasing differential stress in a volume of crust, criteria that involve the paired development of dilation and shear deformation may be most successful.

\subsection{The relationship between strain accumulation and localization}

Tracking the mean and the volume of rock occupied by each strain component in each DVC calculation reveals differences between the strain accumulation and localization processes of the volumetric and shear strains. These processes occur over longer periods of time (i.e. macroscopic axial strain) for the local volumetric strains than the local shear strains. In all but one experiment, both the mean and volume of the dilatational strains exceed those of the contractive strains at some stage of the experiment. In particular, the mean and volume of the local dilation exceed those of the contraction between 0.3 0.7 normalized axial strain, with 1 being the normalized axial strain at failure (Figs 7 and 8). For these experiments, the axial strain at which the mean dilation exceeds the mean contraction occurs close to when the volume occupied by the dilatational strains exceeds the volume occupied by the contractive strains (Figs 7 and 8). In contrast, the timing of when the mean of the left-lateral shear strain exceeds the mean of the right-lateral shear strain coincides with the trends in the volume occupied by the different shear strains over a shorter time period (Figs 7 and 8). Whereas dilation tends to occupy larger volumes (delocalizing) as it increases in amplitude, the shear strain tends to occupy the same or smaller volumes (localizing) as it gains strength (Figs 7, 8 and 11c). Tracking the correlation length of each of the strain components throughout loading supports these observations (Figs 9 and 10). The correlation length of the dilation tends to increase by the largest magnitudes from lower to higher differential stresses, whereas the correlation lengths of the other components tend to change by lower magnitudes (Figs 9 and 10). Throughout loading, the interaction range of dilation tends to increase, while this range tends to decrease or stay similar for the other strain components.

The differences between the evolution of the dilatational and shear strains are somewhat intuitive because dilatational strain involves volume change implicitly, but shear strain does not necessarily. However, the methods of calculating the volume occupied by each strain component and tracking the correlation length do not consider the change in volume produced by each deformation mode. These methods only consider the number of subvolumes that contain the expression of the strain mode, and the magnitude of the strain components in these subvolumes. These analyses demonstrate that the dilatational strain accumulation process involves increases in volume within the subvolume of calculation, increases in the number of subvolumes exhibiting dilation, and increases in the distance of the influence of dilation.

The development of networks of compaction bands may also produce a paired increase in the magnitude of strain accommodated by the bands and the volume occupied by the bands with increasing differential stress. Mair et al. (2000) documented that the total amount of macroscopic axial strain accommodated by high porosity (20 per cent) sandstone in triaxial deformation experiments was linearly related to the number of compaction bands that developed. If the amount of local strain accommodated by each band also increased with the number of bands, then both the magnitude of local strain and volume experiencing this strain would have increased, consistent with the trend observed in the DVC results. Interestingly, this trend does not occur for only the experiments on sandstone, but in the experiments on the other sedimentary rocks and low porosity crystalline rocks as well (Figs 7 and 8). Moreover, this trend may exist for the local contractive strains during compaction band development (e.g. Mair et al. 2000), but the DVC calculations identify this trend in the dilatational strain rather than the contractive strain in 11 of 12 experiments.

The eventual coalescence of many individual fractures into through-going faults produces the observed paired increase in leftor right-lateral shear strain and decrease in the volume occupied by that strain. Fault networks may evolve from distinct fracture segments that propagate until they begin to interact and link with 
neighbouring segments, ultimately forming one or a few larger faults (e.g. Mansfield and Cartwright 2001; Ben-Zion and Sammis 2003; Crider and Peacock 2004; Crider 2015; Peacock et al. 2018). The mean shear strain (in either the left- or right-lateral direction) accommodated by each smaller fault segment at the incipient stages of fault network development may be lower than the mean shear strain accommodated by the more continuous fault strands that develop later. Similarly, the total volume of the incipient fault segments could be higher than the total volume of the through-going fault strands if some of the fault strands are abandoned as other segments link. Under ductile deformation conditions, the magnitude and volume of shear strain accommodated in a shear zone may show a similar behaviour: increasing in magnitude while decreasing in volume. Over time, the effective width of a shear zone may decrease, increasing the shear strain accommodated along the zone, provided the amount the fault-plane parallel displacement remains similar or constant. In both the brittle and ductile realms, the shear strain accumulation and localization processes may evolve in opposite senses: increasing in magnitude while decreasing in volume (e.g. Fig. 11c), as documented in our experiments.

\section{CONCLUSIONS}

Using 12 triaxial deformation X-ray tomography experiments on six rock types, we examine the strain accumulation and localization process as rocks are compressed towards failure. At these experimental conditions representing the upper crust (5-35 MPa confining stress), the strain accumulation process involves the paired increase in several components. The strengths of this paired increase of strain components, as measured with the Pearson correlation coefficient, are moderate-high in all but one experiment (Fig. 4). The correlation between the changes in the dilation and shear strain from one tomogram to the next (or stress step) are stronger than the correlations between the changes in the (1) contraction and shear strain and (2) dilation and contraction. Tracking the volumetric frequency of strain components reveals that the most frequent mode of strain accommodation throughout each complete experiment is the paired increase in the dilation and shear strain in 11 of 12 experiments (Fig. 5). Under low differential stress, the most volumetrically frequent mode of strain accommodation is the paired increase in dilation and contraction (Figs 5, 11b). Under higher differential stress, the most volumetrically frequent mode of strain accommodation is the paired increase in dilation and shear strain (Figs 5, 11b). Tracking the mean of each strain component and volume of rock occupied by the strain component reveals different behaviours of the volumetric and shear strains (Figs 6-8, 11c). Whereas dilation tends to occupy larger volumes (delocalizing) as it gains strength, shear strain tends to occupy the same or smaller volumes (localizing) as it gains strength (Figs 8,11c). Tracking the correlation distance for each of the strain components throughout loading shows that the interaction distance of the dilation grows larger from lower to higher differential stress than any of the other strain components, including the contraction and shear strains (Figs 9-11c). These observations provide constraints on the relevant failure criteria that describe the onset of localized failure, and the corresponding precursory signals. Successful macroscopic failure criteria should incorporate the paired response of dilation and shear strain to describe and predict the evolution towards failure. Future work may use the presented observations of the differing evolutions of magnitude, correlation length and volume occupied by the dilation and shear strain from lower to higher differential stress to assess the success of existing and new failure criteria. The observed trends in strain accumulation and localization tend to be consistent among the six rock types, suggesting that different rock types may not require different failure criteria. This idea suggests that a unified theory may be able to describe precursory strain localization leading to macroscopic failure in rocks of varying microstructure, porosity and composition.

\section{ACKNOWLEDGEMENTS}

The deformation apparatus was built by Sanchez Technology. Elodie Boller, Paul Tafforeau and Alexander Rack provided advice on the design of the tomography setup. Stephen Hall and Erika Tudisco provided advice on the application of TomoWarp2. This study received funding from the Norwegian Research Council (grant 267775). Beamtime was allocated at the European Synchrotron Radiation Facility (Long Term Proposal ES-295). Data storage was provided by UNINETT Sigma2 - the National Infrastructure for High Performance Computing and Data Storage in Norway (project NS9073K). The DVC data are available on UNINET Sigma2 - the National Infrastructure for High Performance Computing and Data Storage in Norway (McBeck 2020). We thank Editor Juan Carlos Afonso, reviewer Hamed Ghaffari and an anonymous reviewer for thorough reviews that helped to improve the manuscript.

\section{REFERENCES}

Aben, F.M., Doan, M.L., Mitchell, T.M., Toussaint, R., Reuschlé, T., Fondriest, M., Gratier, J.-P. \& Renard, F., 2016. Dynamic fracturing by successive coseismic loadings leads to pulverization in active fault zones, $J$. geophys. Res. 121(4), 2338-2360.

Aydin, A. \& Johnson, A. M., 1983. Analysis of faulting in porous sandstones, J. Struct. Geol., 5(1), 19-31.

Ben-Zion, Y. \& Sammis, C. G., 2003. Characterization of Fault Zones, Pure appl. Geophys., 160, 677-715.

Benson, P. M., Thompson, B. D., Meredith, P. G., Vinciguerra, S. \& Young, R. P., 2007. Imaging slow failure in triaxially deformed Etna basalt using 3D acoustic-emission location and X-ray computed tomography, Geophys. Res. Lett., 34, L03303. https://doi.org/10.1029/2006GL028721.

Bourbie, T. \& Zinszner, B., 1985. Hydraulic and acoustic properties as a function of porosity in Fontainebleau sandstone, J. geophys. Res., 90(B13), 11524-11 532.

Brace, W. F., Paulding, B. W. \& Scholz, C. H., 1966. Dilatancy in the fracture of crystalline rocks, J. geophys. Res., 71(16), 3939-3953.

Bridgman, P.W., 1949. Volume changes in the plastic stages of simple compression, J. appl. Phys., 20(12), 1241-1251.

Carbone, D., Aloisi, M., Vinciguerra, S. \& Puglisi, G., 2014. Stress, strain and mass changes at Mount Etna during the period between the 1993-94 and 2001 flank eruptions, Earth-Sci. Rev., 138, 454-468.

Cohen, J. 1988, Statistical Power Analysis for the Behavioural Sciences, 2nd edn, Erlbaum

Cooke, M.L. \& Madden, E.H., 2014. Is the Earth lazy? A review of work minimization in fault evolution, J. Struct. Geol., 66, 334-346.

Coulomb, C., 1776. Sur une application des rêgles maximis et minimis à quelques problèmes de statique, relatives à l'architecture, Acad. Sci. Paris Mem. Math Phys., 7, 343-382.

Crider, J.G., 2015. The initiation of brittle faults in crystalline rock, J. Struct. Geol., 77, 159-174.

Crider, J.G. \& Peacock, D.C., 2004. Initiation of brittle faults in the upper crust: a review of field observations, J. Struct. Geol., 26(4), 691-707.

Crider, J.G. \& Pollard, D.D., 1998. Fault linkage: three-dimensional mechanical interaction between echelon normal faults, J. geophys. Res., 103(B10), 24373-24391.

Frank, F.C., 1965. On dilatancy in relation to seismic sources, Rev. Geophys., 3(4), 485-503. 
Griffith, A. A., 1921. The phenomena of rupture and flow in solids, Phil. Trans. R. Soc. Lond., A, 221(582-593), 163-198.

Hamiel, Y., Liu, Y., Lyakhovsky, V., Ben-Zion, Y. \& Lockner, D., 2004a. A visco-elastic damage model with applications to stable and unstable fracturing, Geophys. J. Int., 159, 1155-1165.

Hamiel, Y., Lyakhovsky, V. \& Agnon, A., 2004b. Coupled evolution of damage and porosity in poroelastic media: theory and applications to deformation of porous rocks, Geophys. J. Int., 156(3), 701-713.

Hamiel, Y., Lyakhovsky, V. \& Agnon, A., 2005. Rock dilation, nonlinear deformation, and pore pressure change under shear, Earth planet. Sci. Lett., 237(3-4), 577-589.

Hamiel, Y, Lyakhovsky, V., Stanchits, S., Dresen, G. \& Ben-Zion, Y., 2009. Brittle deformation and damage-induced seismic wave anisotropy in rocks, Geophys. J. Int., 178, 901-909.

Han, B., Xie, S. Y. \& Shao, J. F., 2016. Experimental investigation on mechanical behavior and permeability evolution of a porous limestone under compression, Rock Mech. Rock Eng., 49(9), 3425-3435.

Handin, J., Hager, R.V., Jr, Friedman, M. \& Feather, J.N., 1963. Experimental deformation of sedimentary rocks under confining pressure: pore pressure tests, AAPG Bull., 47(5), 717-755.

Heap, M. J., Vinciguerra, S. \& Meredith, P., 2009. The evolution of elastic moduli with increasing crack damage during cyclic stressing of a basalt from Mount Etna volcano, Tectonophysics, 471(1-2), 153-160.

Huang, L., Baud, P., Cordonnier, B., Renard, F., Liu, L. \& Wong, T.-F., 2019. Synchrotron X-ray imaging in 4D: multiscale failure and compaction localization in triaxially compressed porous limestone, Earth planet. Sci. Lett., 528, https://doi.org/10.1016/j.epsl.2019.115831.

Inglis, C. E., 1913. Stresses in a plate due to the presence of cracks and sharp corners, Trans. Inst. Naval Archit., 55, 219-241.

Irwin, G. R., 1948, Fracture Dynamics, Fracturing of Metals, American Society of Metal, pp. 147-166.

Kobchenko, M. et al., 2011. 4D imaging of fracturing in organic-rich shales during heating, J. geophys. Res., 116, B12201. https://doi.org/10.1029/20 11JB008565.

Kolosov, G., 1909. Sur le probleme plan dans la théorie d'élasticité, Atti IV Congresso Internationale Matematici, 3, 187-190.

Lawn, B., 1993. Fracture of Brittle Solids, Cambridge University Press.

Lyakhovsky, V., Ben-Zion, Y. \& Agnon, A., 1997. Distributed damage, faulting, and friction, J. geophys. Res., 102(B12), 27635-27649.

Lyakhovsky, V., Hamiel, Y., Ampuero, J.-P. \& Ben-Zion, Y., 2009. Nonlinear damage rheology and wave resonance in rocks, Geophys. J. Int., 178, 910-920.

Lyakhovsky, V., Hamiel, Y. \& Ben-Zion, Y., 2011. A non-local visco-elastic damage model and dynamic fracturing, J. Mech. Phys. Solids, 59(9), 1752-1776.

Mair, K., Main, I. \& Elphick, S., 2000. Sequential growth of deformation bands in the laboratory, J. Struct. Geol., 22(1), 25-42.

Mansfield, C. \& Cartwright, J., 2001. Fault growth by linkage: observations and implications from analogue models, J. Struct. Geol., 23(5), 745-763.

McBeck, J.(2020). The mixology of precursory strain partitioning approaching brittle failure in rocks [Data set], Norstore https://doi.org/10.11582/2020.00002.

McBeck, J.A., Cooke, M.L., Herbert, J.W., Maillot, B. \& Souloumiac, P., 2017. Work optimization predicts accretionary faulting: an integration of physical and numerical experiments, Jgeophys. Res., 122(9), 7485-7505.

McBeck, J. A., Cordonnier, B., Vinciguerra, S. \& Renard, F., 2019. Volumetric and shear strain localization in Mt. Etna basalt, Geophys. Res. Lett., 46(5), 2425-2433.

McBeck, J., Kobchenko, M., Hall, S.A., Tudisco, E., Cordonnier, B., Meakin, P. \& Renard, F., 2018. Investigating the onset of strain localization within anisotropic shale using digital volume correlation of time-resolved X-ray microtomography images, J. geophys. Res., 123 (9), 7509-7528.

Mitra, G., 1994. Strain variation in thrust sheets across the Sevier fold-andthrust belt (Idaho-Utah-Wyoming): implications for section restoration and wedge taper evolution, J. Struct. Geol., 16(4), 585-602.

Mohr, O., 1900. Welche Umstande bedingen die Elastizitatsgrenze und den Bruch eines Materials? Zeit des Ver Deut. Ing., 44:1524-1530.
Moore, D.E. \& Lockner, D.A., 1995. The role of microcracking in shearfracture propagation in granite, J. Struct. Geol., 17(1), 95-114.

Myachkin, V.I., Sobolev, G.A., Dolbilkina, N.A., Morozow, V.N. \& Preobrazensky, V.B., 1972. The study of variations in geophysical fields near focal zones of Kamchatka, Tectonophysics, 14, 287-293.

Nur, A., 1972. Dilatancy, pore fluids, and premonitory variations of $t_{s} / t_{p}$ travel times, Bull. seism. Soc. Am., 62(5), 1217-1222.

Owladeghaffari, H.(2015, Laboratory earthquakes: analysis of fractures and acoustic emission using functional acoustic network, Doctoral dissertation, University of Toronto, Canada.

Paul, B., 1961. A modification of the Coulomb-Mohr theory of fracture, $J$. Appl. Mech., 28(2), 259-268.

Peacock, D.C.P., Sanderson, D.J. \& Rotevatn, A., 2018. Relationships between fractures, J. Struct. Geol., 106, 41-53.

Peng, S. \& Johnson, A.M., 1972. Crack growth and faulting in cylindrical specimens of Chelmsford granite, Int. J. Rock Mech. Min. Sci. Geomech. Abstr., 9(1), 37-86.

Petit, J.P. \& Barquins, M., 1988. Can natural faults propagate under mode II conditions?, Tectonics, 7(6), 1243-1256.

Reches, Z.E. \& Lockner, D.A., 1994. Nucleation and growth of faults in brittle rocks, J. geophys. Res., 99(B9), 18159-18 173.

Renard, F., 2017. Critical evolution of damage towards system size failure in a crystalline rock [Data set], Norstore, https://doi.org/10.11582/2017.00025.

Renard, F., 2018a. Volumetric and shear strain localization in Mt. Etna basalt [Data set], Norstore, https://doi.org/10.11582/2018.00036.

Renard, F., 2018b. Dynamic in situ three-dimensional imaging and digital volume correlation analysis quantify strain localization and fracture coalescence in sandstone [Data set], Norstore, https://doi.org/10.11582/2018.00022.

Renard, F., 2018c. Volumetric and shear processes in crystalline rock during the approach to faulting [Data set], Norstore, https://doi.org/10.11582/2018.00023.

Renard, F., Cordonnier, B., Dysthe, D.K., Boller, E., Tafforeau, P. \& Rack, A., 2016. A deformation rig for synchrotron microtomography studies of geomaterials under conditions down to $10 \mathrm{~km}$ depth in the earth, $J$. Synchrot. Radiat., 23(4), 1030-1034.

Renard, F., Cordonnier, B., Kobchenko, M., Kandula, N., Weiss, J. \& Zhu, W., 2017. Microscale characterization of rupture nucleation unravels precursors to faulting in rocks, Earth planet. Sci. Lett., 476, 69-78.

Renard, F. \& McBeck, J., 2018. Investigating the onset of strain localization within anisotropic shale using digital volume correlation of time-resolved X-ray microtomography images (data set), Norstore, https://doi.org/10.11582/2018.00005.

Renard, F. et al., 2019a. Dynamic in situ three-dimensional imaging and digital volume correlation analysis to quantify strain localization and fracture coalescence in sandstone, Pure appl. Geophys., 176(3), 10831115.

Renard, F., McBeck, J., Kandula, N., Cordonnier, B., Meakin, P. \& Ben-Zion, Y., 2019b. Volumetric and shear processes in crystalline rock approaching faulting, Proc. Natl. Acad. Sci., 116(33), 16234-16239.

Rice, J. R., 1980. The mechanics of earthquake rupture, in Physics of the Earth's Interior, pp. 555-649, eds Dziewonski, A.M. \& Boschi, E., Italian Physical Society.

Rice, J. R. \& Rudnicki, J. W., 1980. A note on some features of the theory of localization of deformation, Int. J. Solids Struct., 16(7), 597-605.

Rodríguez, P. \& Celestino, T. B., 2019. Application of acoustic emission monitoring and signal analysis to the qualitative and quantitative characterization of the fracturing process in rocks, Eng. Fract. Mech., 210, 54-69.

Rudnicki, J. W. \& Rice, J. R., 1975. Conditions for the localization of deformation in pressure-sensitive dilatant materials, J. Mech. Phys. Solids, 23(6), 371-394.

Rutter, E. H. \& Neumann, D. H. K., 1995. Experimental deformation of partially molten Westerly granite under fluid-absent conditions, with implications for the extraction of granitic magmas, J. geophys. Res., 100(B8), $15697-15715$. 
Stanchits, S., Vinciguerra, S. \& Dresen, G., 2006. Ultrasonic velocities, acoustic emission characteristics and crack damage of basalt and granite, Pure appl. Geophys., 163(5-6), 975-994.

Storti, F., Salvini, F. \& McClay, K., 1997. Fault-related folding in sandbox analogue models of thrust wedges, J. Struct. Geol., 19(3-4), 583-602.

Tal, Y., Evans, B. \& Mok, U., 2016. Direct observations of damage during unconfined brittle failure of Carrara marble, J. geophys. Res., 121(3), 1584-1609.

Thompson, B. D., Young, R. P. \& Lockner, D. A.(2005). Observations of premonitory acoustic emission and slip nucleation during a stick slip experiment in smooth faulted Westerly granite, Geophys. Res. Lett., 32(10), https://doi.org/10.1029/2005GL022750.

Tudisco, E., Ando`, E., Cailletaud, R. \& Hall, S.A., 2017. TomoWarp2: a local digital volume correlation code, SoftwareX, 6, 267-270.

Vinciguerra, S., Trovato, C., Meredith, P. G. \& Benson, P. M., 2005. Relating seismic velocities, thermal cracking and permeability in Mt. Etna and Iceland basalts, Int. J. Rock Mech. Min. Sci., 42(7-8), 900-910.

Wong, R.H. \& Chau, K.T., 1998. Crack coalescence in a rock-like material containing two cracks, Int. J. Rock Mech. Min. Sci., 35(2), 147-164.

Wong, T. F., David, C. \& Zhu, W., 1997. The transition from brittle faulting to cataclastic flow in porous sandstones: Mechanical deformation, $J$. geophys. Res., 102(B2), 3009-3025.

Zhao, X.G. \& Cai, M., 2010. A mobilized dilation angle model for rocks, Int. J. Rock Mech. Min. Sci., 47(3), 368-384.

Zhu, W., Baud, P. \& Wong, T.F., 2010. Micromechanics of cataclastic pore collapse in limestone, J. geophys. Res., 115(B4), doi:10.1029/2009JB006610.

\section{SUPPORTING INFORMATION}

Supplementary data are available at $G J I$ online.

Figure S1. Schematic representation of three spatial resolutions used to sample the strain field of a centimeter scale rock sample undergoing deformation. The X-ray tomogram resolution is 6.5 $\mu \mathrm{m}$ per voxel-side length (a). The spatial resolution of the digital volume correlation (DVC) calculation is 20 voxels, or $0.13 \mathrm{~mm}$ per side length (b). The resolution of the sampling grid used to calculate the mean of the strain components within each cube is $0.2 \mathrm{~mm}$ (c). Grey lines in (a) are not to scale relative to the lines in (b-c). Lines in (b) and (c) are the correct approximate scale.

Figure S2. Relationship between the magnitudes of the strain component means for experiments on monzonite (a), limestone (b) and shale (c). Normalized dilation versus normalized contraction, normalized shear strain versus normalized contraction, and normalized shear strain versus normalized dilation shown in left-hand, middle and right-hand columns, respectively. The strain populations are normalized by the maximum value of the population. Red lines show the linear regression through the pairs of strain magnitudes.

Figure S3. Examining the spatial coincidence of the change in the mean strain magnitude throughout each complete experiment (a), in the first DVC calculation (b) and in the final DVC calculation (c). Bars indicate the strain accommodation mode: the number of voxels in which the individual strain component or pair of components increased from one DVC calculation to the next out of the total number of voxels in the experiment (a) or in the DVC calculation $(b, c)$. The ratios that report increases in the individual strain modes do not include the voxels in which multiple strain modes increase.

Figure S4. Evolution of strain accommodation throughout time (macroscopic axial strain) in experiments on (a) sandstone, (b) basalt, (c) monzonite, (d) granite, (e) shale and (f) limestone. First row of each subsection shows the mean of the strain components, including the dilation, contraction, left-lateral and right-lateral shear strain. Second row shows the volume of the core that each strain component occupied. Left- and right-hand columns of each subsection show the dilation and contraction, and left- and right-lateral shear strain, respectively. Blue and green rectangles highlight the time when the dilation mean (blue) or volume (green) exceed the contraction mean or volume, and when the left-lateral shear strain mean or volume exceed the right-lateral shear strain mean or volume, respectively.

Figure S5. Distribution of the correlation coefficient with increasing distance for five strain components at each stress step. The shear strain includes both the left- and right-lateral shear strain. Colours of the lines indicate the differential stress of the DVC calculations, from lower (blue) to higher (yellow). Triangles show the distance when the correlation coefficient decreases from higher values (0.5), indicative of strong correlations, to lower values, indicative of moderate weak correlations. We consider this distance as the correlation length.

Please note: Oxford University Press is not responsible for the content or functionality of any supporting materials supplied by the authors. Any queries (other than missing material) should be directed to the corresponding author for the paper. 